\title{
BICONTINUITY OF THE DIXMIER MAP
}

\author{
OLIVIER MATHIEU
}

1. Notations, generalities, conventions

2. Polarizations

3. Semicontinuity principle

4. The orbital correspondence

5. Going up lemmas

6. Proof of the Theorem

\section{INTRODUCTION}

Let $\mathfrak{g}$ be a solvable Lie algebra over $\mathbf{C}$ and let $\mathscr{A}$ be its algebraic adjoint group. The Dixmier map $I$ is a bijective and continuous correspondence between the set $\mathfrak{g}^{*} / \mathscr{A}$ of coadjoint orbits and the set Prim $\mathfrak{g}$ of all the primitive ideals of $U(\mathfrak{g})$. Actually the map $I$ (which is the infinitesimal analog of the Kirillov orbits method [K, AK], has been defined by J. Dixmier [Di $\left.{ }_{1}, \mathrm{Di}_{2}\right]$, the continuity of $I$ has been proved by N. Berline-Conze, M. Vergne, and M. Duflo $[C V, C D]$, the surjectivity by $M$. Duflo $\left[\mathrm{Du}_{1}, \mathrm{Du}_{2}\right]$ and the injectivity by $\mathrm{R}$. Rentschler [R, BGR] (Dixmier's book [ $\left.\mathrm{Di}_{3}\right]$ contains proofs of all these results). We prove that $I$ is bicontinuous, i.e., that the inverse of $I$ is continuous.

Theorem. The map I is bicontinuous.

The proof of the Theorem uses in an essential way finite characteristics tools, namely the representation theory of restricted algebras. More precisely the proof is based on (what seems to be) a new operation on coadjoint orbits, that we call orbital correspondence. Roughly speaking the orbital correspondence is a canonical way to linearize Cartier's operators [Ca], up to the $G$-action (this linearization is very different from the Deligne and Illusie linearization of Cartier's operators [DI]).

Let $\mathfrak{g}$ be the Lie algebra of an algebraic solvable group $G$ over a field $k$ of finite characteristic. By the orbital correspondence we associate to any $G$-orbit $\mathscr{O}$ of $\mathfrak{g}^{*}$ an orbit $C \mathscr{O}$ of the space $\left(F_{*} \mathfrak{g}\right)^{*}$ of the $p$-linear map of $\mathfrak{g}$. It turns out that this correspondence has a simple interpretation in terms of the orbits method. Actually, by using the central characters any prime ideal $K$ of $U(\mathfrak{g})$ gives rise to a subvariety $m(K)$ of $\left(F_{*} \mathfrak{g}\right)^{*}$. Then we prove that $m(I(\mathscr{O}))$ is

Received by the editors March 28, 1991.

1991 Mathematics Subject Classification. Primary 17B35, 17B30, 17 B50. 
exactly $\overline{C \mathscr{O}}$. The proof of the main result crucially uses a theorem of Weisfeiler and $\mathrm{Kac}[\mathrm{WF}]$. Indeed a similar idea has been used in $\left[\mathrm{M}_{2}\right]$.

Note that for nilpotent Lie algebras the bicontinuity of $I$ is due to N. BerlineConze [C]. In [R], R. Rentschler defines a stratification of $\mathfrak{g}^{*} / \mathscr{A}$ and Prim $U(\mathfrak{g})$ and he proves that the restriction of $I$ to each stratum is bicontinuous. The orbital correspondence allows us to compare the behavior of $I$ on different strata. The Theorem was an open problem of $\left[\mathrm{Di}_{3}\right]$.

\section{Notations, GENERALITIES, CONVENTIONS}

(1.1) All fieds involved in the paper have characteristic 0 or $p \neq 2$. Here varieties are not necessarily irreducible. Unless explicitly stated, subvarieties are closed. If $X$ is an affine scheme over a ring $k$, the ring of regular functions over $X$ is denoted by $k[X]$.

If $V$ is a vector space over a perfect field $k$ of characteristic $p \neq 0$, we will denote by $F_{*} V$ the Frobenius twist of $V$. More precisely, $F_{*} V$ is the $k$-vector space with the same underlying group structure, and the scalar multiplication by $x$ in $F_{*} V$ corresponds to the scalar multiplication by $x^{1 / p}$ in $V$. Thus $\left(F_{*} V\right)^{*}$ is the space of $p$-linear maps on $V$.

(1.2) Let $k$ be a field. All Lie algebras considered here have finite dimension over $k$. Let $\mathfrak{g}$ be a Lie algebra. The weights of $\mathfrak{g}$ are linear forms $\lambda \in \mathfrak{g}^{*}$ such that $\lambda([\mathfrak{g}, \mathfrak{g}])=0$. Any weight $\lambda$ of $\mathfrak{g}$ determines a representation of dimension one. This representation will also be denoted by $\lambda$. The weights of $\mathfrak{g}$ corresponding to one-dimensional subquotients of the adjoint representation are called the roots.

Let $\mathfrak{p}$ be a subalgebra of $\mathfrak{g}$, and let $\lambda$ be a weight of $\mathfrak{p}$. We will denote by $\operatorname{ind}(\mathfrak{p}, \mathfrak{g}) \lambda$ the induced module. For any $x \in \mathfrak{p}$ we set $\lambda^{*}(x)=\lambda(x)+$ $\frac{1}{2} \operatorname{tr}(\operatorname{ad}(x) \mid \mathfrak{g} / \mathfrak{p})$, and we set $\operatorname{ind}^{*}(\mathfrak{p}, \mathfrak{g}) \lambda=\operatorname{ind}(\mathfrak{p}, \mathfrak{g}) \lambda^{*}$. The module ind ${ }^{*}(\mathfrak{p}, \mathfrak{g}) \lambda$ is called the twisted induced module.

More generally, let $A$ be a commutative ring, let $\mathfrak{g}$ be a Lie algebra over $A$, let $\mathfrak{p}$ be a subalgebra, and let $\lambda: \mathfrak{p} \rightarrow A$ be a weight of $\mathfrak{p}$ (i.e., $\lambda$ is $A$-linear and it satisfies $\lambda([\mathfrak{p}, \mathfrak{p}])=0)$. Then, if $\mathfrak{g} / \mathfrak{p}$ is a finite rank projective $A$-module and if 2 is invertible in $A$, we can define the twisted induced module $\operatorname{ind}^{*}(\mathfrak{p}, \mathfrak{g}) \lambda$.

(1.3) Let $k$ be an algebraically closed field of characteristic zero. An algebraic solvable Lie algebra is a pair $(\mathfrak{g}, G)$, where $\mathfrak{g}$ is a solvable Lie algebra and $G$ is a connected solvable algebraic group with Lie algebra $\mathfrak{g}$ such that the Lie algebra of the unipotent radical of $G$ is the unipotent radical of $\mathfrak{g}$. The following lemma is well known (actually it is a particular and simple case of Chevalley's Replicas Theory or Grothendieck's Tannaka Theory).

Lemma 1.3.1. Let $\mathfrak{g}$ be a solvable Lie algebra and let $\mathscr{A}$ be its adjoint group. Then there exists an algebraic solvable Lie algebra $\mathfrak{g}$ ' with group $G^{\prime}$ containing $\mathfrak{g}$ such that

(1) $\mathfrak{g}$ is an ideal of $\mathfrak{g}^{\prime}$, 
(2) any $\mathscr{A}$-stable subset of $\mathfrak{g}^{*}$ is $G^{\prime}$-stable, and

(3) any ad $(\mathfrak{g})$-submodule of $U(\mathfrak{g})$ is stable by $\operatorname{ad}\left(\mathfrak{g}^{\prime}\right)$.

Proof. Let $Q$ be the subgroup of $\mathfrak{g}^{*}$ generated by the roots of $\mathfrak{g}$. By Ado's Theorem there exists a faithful $\mathfrak{g}$-module $M$ of finite dimension such that all simple subquotients of $M$ are weights in $Q$. Moreover, we can assume that $M$ contains the adjoint representation as direct summand. Let $\mathfrak{g}^{\prime}$ (respectively $\Gamma$ ) be the set of elements $x \in \operatorname{End}(M)$ (respectively in $\operatorname{GL}(M)$ ) such that, for any integers $n$ and $m, x$ stabilizes any $\mathfrak{g}$-submodule of $M^{\otimes m} \otimes\left(M^{*}\right)^{\otimes n}$ and acts as zero (respectively as 1) on every trivial subquotient of $M^{\otimes m} \otimes\left(M^{*}\right)^{\otimes n}$. Let $G^{\prime}$ be the connected component of $\Gamma$ (actually it is easy to see that $\Gamma$ is connected). Then it is clear that $\left(\mathfrak{g}^{\prime}, G^{\prime}\right)$ is a convenient choice. Q.E.D.

(1.4) Let $k$ be an algebraically closed field of characteristic zero, and let $(\mathfrak{g}, G)$ be an algebraic solvable Lie algebra. Let $\mathfrak{u}$ be the nilpotent radical of $\mathfrak{g}$, let $U$ be the unipotent radical of $G$, and let $l$ be an integer such that $\operatorname{ad}\left(x_{1}\right) \circ \cdots \circ \operatorname{ad}\left(x_{l}\right)=0$ for any $x_{1}, \ldots, x_{l} \in \mathfrak{u}$. It is well known that there exists a map $\exp : \mathfrak{u} \rightarrow U$ which commutes with $\operatorname{Ad}(G)$ such that

(1.4.1) the differential of exp at 0 is the identity,

(1.4.2) $\operatorname{Ad}(\exp x) \cdot g=\sum_{0 \leq i<l}(1 / i !) \operatorname{ad}^{i}(x)(g)$, and

(1.4.3) $(\exp x)(\exp y)=\exp \left(\sum_{1 \leq i \leq l} H_{i}(x, y)\right)$

for any $x, y \in \mathfrak{u}$ and $g \in \mathfrak{g}$, where $H_{i}$ is the homogenous term of degree $i$ of the Campbell-Hausdorff series. Moreover, $\exp : \mathfrak{u} \rightarrow U$ is bijective. Up to a denominator $d_{i}, H_{i}$ is a Lie polynomial over $\mathbf{Z}$ in $x$ and $y$, and all primes involved in the denominator $d_{i}$ are $\leq i$. This explains the following definition of algebraicity of a solvable Lie algebra over fields of nonzero characteristic.

(1.5) Let $k$ be an algebraically closed field of characteristic $p \neq 0$. By definition an algebraic solvable Lie algebra will be a pair $(\mathfrak{g}, G)$ which satisfies the three conditions (1.5.1), (1.5.2), and (1.5.3) stated below.

(1.5.1) $G$ is a smooth connected solvable algebraic group whose Lie algebra is $\mathfrak{g}$ (actually the hypothesis " $G$ is smooth" is equivalent to " $G$ is reduced"). It acts as the automorphism group of $\mathfrak{g}$ and the differential of the action Ad is ad.

(1.5.2) The Lie algebra of the unipotent radical $U$ of $G$ is the nilpotent radical $\mathfrak{u}$ of $\mathfrak{g}$ and there is a given map $\exp : \mathfrak{u} \rightarrow U$ which commutes with $\operatorname{Ad}(G)$ such that

(1.5.2.1) the differential of $\exp$ at 0 is the identity,

(1.5.2.2) $\operatorname{Ad}(\exp x) \cdot g=\sum_{0<i<p-1}(1 / i !) \operatorname{ad}^{i}(x)(g)$, and

(1.5.2.3) $\exp x \exp y=\exp \left(\sum_{1<i \leq p-1} H_{i}(x, y)\right)$

for any $x, y \in \mathfrak{u}$ and $g \in \mathfrak{g}$. It follows that $\exp : \mathfrak{u} \rightarrow U$ is bijective and for any commuting elements $x, y \in \mathfrak{u}$ we have $(\exp x)(\exp y)=\exp (x+y)$. Actually it is not necessary to assume that $\operatorname{ad}\left(x_{1}\right) \circ \cdots \circ \operatorname{ad}\left(x_{p}\right)=0$ for any $x_{1}, \ldots, x_{p} \in \mathfrak{u}$ but it is likely a provision of the previous three such conditions.

(1.5.3) Let $Q(\mathfrak{g})$ be the subgroup of $\mathfrak{g}^{*}$ generated by the roots of $\mathfrak{g}$. Similarly 
the characters of $G$ involved in the simple subquotients of the adjoint action Ad are called the roots of $G$, and let $Q(G)$ be the lattice generated by the roots of $G$. Let $d: Q(G) \rightarrow Q(\mathfrak{g})$ be the differential. We require that for any set $A$ of the roots of $G$ the rank of the lattice generated by $A$ is the same as the dimension of the $\mathbf{F}_{p}$-vector space generated by $d(A)$.

(1.6) Let $k$ be an algebraically closed field of characteristic $p \neq 0$ and let $(\mathfrak{g}, G)$ be an algebraic solvable Lie algebra. Identify $\mathfrak{g}$ with the right invariant derivations of $k[G]$. Then for any $x \in \mathfrak{g}, x^{p}$ is also a right invariant derivation of $k[G]$. Denote by $x^{(p)}$ the corresponding element of $\mathfrak{g}$. Thus the map $x \mapsto x^{(p)}$ defines a $G$-invariant $p$-structure on $\mathfrak{g}$.

Lemma 1.6.1. For any $x$ in the unipotent radical of $\mathfrak{g}$, we have $x^{(p)}=0$.

Proof. Let $L$ be the left action of $G$ on $k[G]$. Let $f \in k[G]$ and $t \in k$. Then we have $(L(\exp t x)-\mathrm{Id}) f=t x \cdot f+\sum_{i \geq 2} t^{i} f_{i}$, where the $f_{i}$ belong to $k[G]$ and almost all the $f_{i}$ are zero. So for any $n \geq 1$ we have $(L(\exp t x)-\operatorname{Id})^{n} f=$ $t^{n} x^{n} \cdot f+\sum_{i \geq n+1} t^{i} f_{i, n}$, where the $f_{i, n}$ belong to $k[G]$ and almost all the $f_{i, n}$ are zero. We have $(L(\exp t x)-\mathrm{Id})^{p}=L(\exp p t x)-\mathrm{Id}=0$. So we get $x^{(p)}=0$. Q.E.D.

For any $\lambda \in \mathfrak{g}^{*}$ let $\mathfrak{g}(\lambda)$ be the stabilizer of $\lambda$ in $G$ and let $G(\lambda)$ be the connected component of the stabilizer of $\lambda$ in $G$. The following lemma follows easily from conditions (1.5.2) and (1.5.3).

Lemma 1.6.2. For any $\lambda$, the group $G(\lambda)$ is smooth and its Lie algebra is $\mathfrak{g}(\lambda)$.

(1.7) Recall a few facts from the theory of restricted algebras (general reference books are $[\mathrm{J}, \mathrm{SF}])$. Let $k$ be an algebraically closed field of finite characteristic $p$ and let $\mathfrak{g}$ be a restricted Lie algebra. The elements of $\left(F_{*} \mathfrak{g}\right)^{*}$ will be called central characters. For any $x \in \mathfrak{g}$, set $\sigma(x)=x^{p}-x^{(p)}$. Then $\sigma(x)$ is a central element of $U(\mathfrak{g})$ and the map $\sigma$ is $p$-linear. The central algebra generated by $\sigma(\mathfrak{g})$ will be denoted by $O(\mathfrak{g})$ and it is canonically isomorphic to $S\left(F_{*} \mathfrak{g}\right)$. Thus any central character of $\mathfrak{g}$ corresponds with a character of $O(\mathfrak{g})$.

(1.8) Let $(\mathfrak{g}, G)$ be an algebraic solvable Lie algebra. The subgroup of $\mathfrak{g}^{*}$ generated by the roots will be denoted by $Q(\mathfrak{g})$ and the unipotent radical of $\mathfrak{g}$ by $\mathfrak{u}$. For any subalgebra $\mathfrak{a}$ of $\mathfrak{g}$ we will denote by $Q(\mathfrak{a})$ the restriction of $Q(\mathfrak{g})$ to $\mathfrak{a}$. The set of weights of $\mathfrak{a}$ is denoted $X(\mathfrak{a})$. We also set $\mathfrak{u}(\mathfrak{a})=\mathfrak{u} \cap \mathfrak{a}$ and $\mathbf{U}(\mathfrak{a})=\exp (\mathfrak{u}(\mathfrak{a}))$. We say that $\mathfrak{a}$ is algebraic in $\mathfrak{g}$ if $\mathfrak{a}$ is the Lie algebra of some smooth closed subgoup of $G$.

When the characteristic of $k$ is prime the algebraic subalgebras are exactly the restricted subalgebras. A central character $E$ of $\mathfrak{a}$ such that $E([\mathfrak{a}, \mathfrak{a}])=0$ is called a central weight. The set of all the central weights of $\mathfrak{a}$ is denoted by $X_{F}(\mathfrak{a})$.

In the paper we will always fix a $G$-invariant flag $\mathscr{F}: 0=\mathfrak{g}_{0} \subset \mathfrak{g}_{1} \subset \cdots$ of $\mathfrak{g}$ such that each element $\mathfrak{g}_{i}$ of $\mathscr{F}$ is an algebraic ideal of $\mathfrak{g}$ of dimension $i$. 
Moreover, we require that $\mathfrak{u}$ be one of the elements of $\mathscr{F}$.

(1.9) Let $A$ be an associative algebra. Unless stated otherwise an ideal of $A$ will be a two-sided ideal. The set of prime ideals is denoted by $\operatorname{Spec} A$. Similarly let $X$ be a variety. By an $\mathscr{O}_{X}$-algebra we mean an associative quasicoherent $\mathscr{O}_{X}$-algebra and by an ideal of an $\mathscr{O}_{X}$-algebra we mean a quasi-coherent two-sided ideal. The set of prime ideals of an $\mathscr{O}_{X}$-algebra $\mathscr{D}$ will be denoted by $\operatorname{Spec} \mathscr{D}$.

(1.10) Let $X \subset \prod_{i \in I} X_{i}$ be a subset in a product of sets. As usual we will denote by $p_{i}$ (respectively $p_{i, j}$ ) the projection on the $i$ th factor (respectively on $\left.X_{i} \times X_{j}\right)$.

\section{Polarizations}

Let $k$ be an algebraically closed field. Let $V$ be the vector space of finite dimension $n$, let $X$ be a variety, and let $B$ be a skew-bilinear map on the vector bundle $V \times X$ over $X$. Thus for any point $x \in X$ we have a skewbilinear form $B(x)$ on $V$ and the map $x \rightarrow B(x)$ is a regular map fom $X$ to $\Lambda^{2} V^{*}$. Fix $0=V_{0} \subset V_{1} \subset \cdots \subset V_{n}=V$ be a flag in $V$. For any $i$ with $1 \leq i \leq n$ let $A_{i}(x)$ be the kernel of $B(x) \mid V_{i}$ and set $p(x)=\sum_{1 \leq i \leq n} A_{i}(x)$. Thus $p(x)$ is a maximal isotropic subpace for $B(x)$ (see Vergne [ $[\overline{\mathrm{B}}$, Chapter IV, Proposition 1.1.2]). Its dimension is called the index of $B(x)$ and it is denoted by ind $(x)$. Let $Y$ be an irreducible component of $X$. The set $y \in Y$ with minimal index is an open subset $Y_{\text {reg }}$. By definition the index of $Y$ is the index of any element of $Y_{\text {reg }}$. We say that $X$ has pure index $d$ if the index of any irreducible component of $X$ is $d$. When $X$ has pure index $d$ we set $X_{\text {reg }}=\{x \in X \mid$ ind $(x)=d\}$.

Assume that $X$ has pure index $d$. Then there exists a dense open subset $X^{0}$ of $X_{\text {reg }}$ on which the map $x \in X^{0} \rightarrow p(x) \in$ Grass $_{d} V$ is a regular morphism. Let $X_{\text {reg }}^{0}$ be the maximal such open set. Thus define $\pi: X^{0} \rightarrow\left(\operatorname{Grass}_{d} V\right) \times X$ by the formula $\pi(x)=(p(x), x)$. The closure of $\pi\left(X^{0}\right)$ in (Grass $\left._{d} V\right) \times X$ does not depend on a particular choice of $X^{0}$. This variety will be denoted by $\Sigma(X)$.

Lemma 2.1. The projection $p_{2}: \Sigma(X) \rightarrow X$ is a surjective and projective morphism.

Proof. As Grass ${ }_{d} V$ is a projective variety, the morphism $p_{2}$ is projective. It is surjective because its image is closed and contains $X_{\text {reg }}^{0}$. Q.E.D.

Let $\mathfrak{g}$ be an algebraic solvable Lie algebra and let $\mathscr{F}$ be a flag of $\mathfrak{g}$ as in (1.8). Then there is associated to any $\lambda \in \mathfrak{g}^{*}$ a skew-bilinear form $B(\lambda)$ on $\mathfrak{g}$ defined by the formula $B(\lambda)(x, y)=\lambda([x, y])$. As usual we denote by $\mathfrak{g}(\lambda)$ the kernel of $B(\lambda)$, i.e., the stabilizer of $\lambda$. By definition a polarization at $\lambda$ is a Lie subalgebra which is a maximal isotropic subspace of $B(\lambda)$. A polarization $\mathfrak{p}$ is 
called a Pukhansky polarization if we have $\mathfrak{p} \subset \mathfrak{g}(\lambda)+\mathfrak{u}$. The maximal isotropic subpace $\mathfrak{p}(\lambda)$ (as previously defined) is a polarization (Vergne [B, Chapter IV, Proposition 1.1.2]). It is called the Vergne polarization.

When $k$ has finite characteristic $p, \mathfrak{p}(\lambda)$ is restricted because it is naturally defined as a sum of restricted subalgebras. Moreover, any central character $E$ of $\mathfrak{g}$ can be seen as a linear form of the $F_{*} \mathfrak{g}$. Thus we can also define the associated skew-bilinear form $B(E)$ and its Vergne polarization $\mathfrak{p}(E)$ and the results of this section stated for linear forms remain true for central characters.

The following lemma is well known.

Lemma 2.2. Let $\lambda, \mu \in \mathfrak{g}^{*}$.

(2.2.1) The Vergne polarization $\mathfrak{p}(\lambda)$ is a Pukhansky polarization.

(2.2.2) Let $\mathfrak{p}$ be a Pukhansky polarization at $\lambda$. If $\lambda-\mu \mid \mathfrak{p}=0$, then $\mu$ is in the $\mathrm{U}(\mathfrak{p})$-orbit of $\lambda$. Moreover, if $\mathfrak{p}$ is the Vergne polarization at $\lambda$, then $\mathfrak{p}$ is also the Vergne polarization at $\mu$.

Proof. (2.2.1) Let $\mathfrak{t}$ be any torus of $\mathfrak{p}(\lambda)$ of dimension 1. Set $\mathfrak{g}^{\prime}=\mathfrak{t} \oplus \mathfrak{u}$ and let $\lambda^{\prime}$ and $\lambda^{\prime \prime}$ be the restrictions of $\lambda$ to $\mathfrak{g}^{\prime}$ and $\mathfrak{u}$, respectively. As $\mathfrak{u}$ is an element of the flag $\mathscr{F}, \mathfrak{u}(\mathfrak{p}(\lambda))$ is a polarization at $\lambda^{\prime \prime}$. Moreover, $\mathfrak{t} \oplus \mathfrak{u}(\mathfrak{p}(\lambda))$ is an isotropic subspace of $B\left(\lambda^{\prime}\right)$. Hence $B\left(\lambda^{\prime}\right)$ and $B\left(\lambda^{\prime \prime}\right)$ have the same rank. Thus we have $\mathfrak{g}^{\prime}\left(\lambda^{\prime}\right) \not \subset \mathfrak{u}$. Let $\mathfrak{t}^{\prime}$ be a maximal torus of $\mathfrak{g}^{\prime}\left(\lambda^{\prime}\right)$. We have $\mathfrak{t}=\mathfrak{t}^{\prime}$ modulo $\mathfrak{u}$. As $\mathfrak{t}^{\prime}$ is contained in some maximal torus of $\mathfrak{g}$ we have $\left[\mathfrak{t}^{\prime}, \mathfrak{g}\right]=\left[\mathfrak{t}^{\prime}, \mathfrak{u}\right]$. So we have $\mathfrak{t}^{\prime} \subset \mathfrak{g}(\lambda)$, and $\mathfrak{t} \subset \mathfrak{g}(\lambda)+\mathfrak{u}$. As $\mathfrak{t}$ is arbitrary, we get the first assertion.

(2.2.2) Set $\mathfrak{u}(\lambda)=\mathfrak{g}(\lambda) \cap \mathfrak{u}$ and let $2 r$ be the rank of $B(\lambda)$. Set $Z=\lambda+\mathfrak{p}^{\perp}$. We have $\operatorname{dim}(\mathfrak{u}(\mathfrak{p}) / \mathfrak{u}(\lambda))=r$. As $\mathbf{U}(\mathfrak{p}) \cdot \lambda$ is a closed subvariety of $Z$ with the same dimension, we have $Z=\mathbf{U}(\mathfrak{p}) \cdot \lambda$. So we have $\mu=\operatorname{Ad}(u)(\lambda)$ for some $u \in \mathbf{U}(\mathfrak{p})$. Moreover, if $\mathfrak{p}$ is the Vergne polarization at $\lambda$ we have $\mathfrak{p}(\mu)=\operatorname{Ad}(u)(\mathfrak{p}(\lambda))=\mathfrak{p}(\lambda)$. Q.E.D.

Let $\tau: \Sigma \rightarrow \Theta$ be a morphism of varieties. A subset $S$ of $\Sigma$ is called saturated (or $\tau$-saturated) if we have $S=\tau^{-1}(\tau(S))$. The morphism $\tau$ is called locally trivializable if any point $y \in \Theta$ admits an open neighborhood $U$ such that $\tau^{-1}(U)$ is isomorphic to $U \times \tau^{-1}(y)$ and $\tau$ is conjugated to the first projection. The following lemma is obvious.

Lemma 2.3. Let $\tau: \Sigma \rightarrow \Theta$ be a locally trivializable morphism of varieties. Let $U$ be a locally closed saturated subset of $\Sigma$. Then $\tau(U)$ is locally closed. Moreover, $\bar{U}$ is saturated and $\tau(\bar{U})=\overline{\tau(U)}$.

Let $d$ be an integer. Let $P_{d}^{*} \mathfrak{g}$ be the vector bundle over Grass ${ }_{d} \mathfrak{g}$ whose fiber over any point $E$ is $E^{*}$ and which is a quotient of the trivial bundle Grass $_{d} \mathfrak{g} \times \mathfrak{g}^{*}$, and let $\tau:$ Grass $_{d} \mathfrak{g} \times \mathfrak{g}^{*} \rightarrow P_{d}^{*} \mathfrak{g}$ be the quotient map. Let $X$ be a locally closed $G$-invariant subvariety of $\mathfrak{g}^{*}$ with pure index $d$. Set $\Theta(X)=\tau(\Sigma(X))$.

Lemma 2.4. Assume that $X$ is closed in $\mathfrak{g}^{*}$. 
(2.4.1) The set $\Theta(X)$ is closed in $P_{d}^{*} \mathfrak{g}$.

(2.4.2) Let $(\mathfrak{a}, \mu)$ be in $\Sigma(X)$ and $\lambda \in \mathfrak{g}^{*}$. Assume that the restriction of $\lambda-\mu$ to $\mathfrak{a}$ vanishes. Then $(\mathfrak{a}, \lambda)$ belongs to $\Sigma(X)$ and $\lambda$ belongs to $X$.

Proof. First note that the morphism $\tau: \mathfrak{g}^{*} \times$ Grass $_{d} \rightarrow P_{d}^{*} \mathfrak{g}$ is locally trivializable. By Lemma 2.2, $\Sigma\left(X_{\text {reg }}^{0}\right)$ is $\tau$-saturated. So, by Lemma 2.3, $\Theta(X)$ is closed and $\Sigma(X)$ is saturated. This proves the lemma. Q.E.D.

Let $\lambda \in \mathfrak{g}^{*}$. For any subalgebra $\mathfrak{b}$, we denote by $\mathfrak{b}(\lambda)$ the stabilizer of $\lambda \mid \mathfrak{b}$. We say that an algebraic subalgebra $\mathfrak{b}$ is a Pukhansky subalgebra if $\mathfrak{g}(\lambda) \subset$ $\mathfrak{b}(\lambda) \subset \mathfrak{g}(\lambda)+\mathfrak{u}$. Thus a Pukhansky polarization is a polarization which is a Pukhansky algebra. Let $\mathscr{X}(\lambda)$ be the set of pairs $(\mathfrak{a}, \mathfrak{b})$ of subalgebras of $\mathfrak{g}$ such that $\mathfrak{a}$ is algebraic and isotropic, $\mathfrak{b}$ is a coisotropic Pukhansky algebra, and $\mathfrak{b}(\lambda) \subset \mathfrak{a} \subset \mathfrak{b}$.

Let $(\mathfrak{a}, \mathfrak{b}) \in \mathscr{X}(\lambda)$. Let $\mathfrak{c}$ be the maximal ideal of $\mathfrak{b}$ such that $\lambda(\mathfrak{c})=0$ and $\mathfrak{c} \subset \mathfrak{u}(\mathfrak{a})$. Now assume that $\mathfrak{a} \neq \mathfrak{b}$. We will describe eight moves to get a new element $\left(\mathfrak{a}^{\prime}, \mathfrak{b}^{\prime}\right)$ in $\mathscr{X}(\lambda)$ such that $\operatorname{dim} \mathfrak{b} / \mathfrak{a}=\operatorname{dim} \mathfrak{b}^{\prime} / \mathfrak{a}^{\prime}+1$.

(2.5.1) Assume that there exists a subspace $\mathfrak{d}$ with $\mathfrak{c} \subset \mathfrak{d} \subset \mathfrak{u}(\mathfrak{b})$ such that $\mathfrak{d} / \mathfrak{c}$ is a one-dimensional noncentral ideal of $\mathfrak{b} / \mathfrak{c}$. Choose $\alpha \in Q(\mathfrak{g})$ such that $\operatorname{Ad}(b \mid \mathfrak{d} / \mathfrak{c})=\alpha(b)$ for any $b \in \mathfrak{b}$.

Move (1.a). Assume $\alpha(\mathfrak{a})=0$ and $\mathfrak{d} \subset \mathfrak{a}$. Then we set $\mathfrak{b}^{\prime}=\operatorname{Ker} \alpha \mid \mathfrak{b}$ and $\mathfrak{a}^{\prime}=\mathfrak{a}$. It follows from the hypothesis that we have $\lambda(\mathfrak{d}) \neq 0$. So we have $\mathfrak{b}^{\prime}(\lambda)=\mathfrak{b}(\lambda)+\mathfrak{d}$. Hence $\mathfrak{b}^{\prime}$ is a Pukhansky subalgebra.

Move (1.b). Assume $\alpha(\mathfrak{a})=0$ and $\mathfrak{d} \not \subset \mathfrak{a}$. Then we set $\mathfrak{b}^{\prime}=\mathfrak{b}$ and $\mathfrak{a}^{\prime}=\mathfrak{a}+\mathfrak{d}$. Note that we have $\alpha(\mathfrak{g}(\lambda))=0$.

Move (1.c). Assume $\alpha(\mathfrak{a}) \neq 0$ and $\lambda(\mathfrak{d})=0$. Then we have $\mathfrak{d} \not \subset \mathfrak{a}$. Set $\mathfrak{b}^{\prime}=\mathfrak{b}$ and $\mathfrak{a}^{\prime}=\mathfrak{a}+\mathfrak{d}$.

Move (1.d). Assume $\alpha(\mathfrak{a}) \neq 0$ and $\lambda(\mathfrak{d}) \neq 0$. Then we have $\mathfrak{d} \not \subset \mathfrak{a}$. Set $\mathfrak{b}^{\prime}=\operatorname{Ker} \alpha \mid \mathfrak{b}$ and $\mathfrak{a}^{\prime}=\mathfrak{a} \cap \mathfrak{b}^{\prime}+\mathfrak{d}$. Note that we have $\alpha(\mathfrak{g}(\lambda))=0$. Moreover, we have $\mathfrak{b}^{\prime}(\lambda)=\mathfrak{b}(\lambda)+\mathfrak{d}$. Hence $\mathfrak{b}^{\prime}$ is a Pukhansky subalgebra.

(2.5.2) Assume that there exists a subspace $\mathfrak{z}$ with $\mathfrak{c} \subset \mathfrak{z} \subset \mathfrak{u}(\mathfrak{b})$ such that $\mathfrak{z} / \mathfrak{c}$ is a one-dimensional central ideal of $\mathfrak{b} / \mathfrak{c}$. Moreover, assume $\mathfrak{z} \not \subset \mathfrak{a}$.

Move (2). Set $\mathfrak{b}^{\prime}=\mathfrak{b}$ and $\mathfrak{a}^{\prime}=\mathfrak{a}+\mathfrak{z}$.

(2.5.3) Assume that there exists a subspace $\mathfrak{t}$ with $\mathfrak{c} \subset \mathfrak{t} \subset \mathfrak{u}(\mathfrak{b})$ such that $\mathfrak{t}=\mathfrak{c} \oplus k \cdot y \oplus k \cdot z$. Moreover, we assume that $\lambda(y)=0, \lambda(z)=1, z \in \mathfrak{a}, z+\mathfrak{c}$ is central in $\mathfrak{b} / \mathfrak{c}$, and $[b, y]=\alpha(b) y+\beta(b) z$ (modulo $\mathfrak{c}$ ) for any $b \in \mathfrak{b}$ for some $\alpha \in Q(\mathfrak{g}), \beta \in \mathfrak{b}^{*}-\{0\}$. When $\alpha \mid \mathfrak{b}$ is not zero, we assume that $\alpha \mid \mathfrak{b}$ and $\beta$ are not proportional. Let $\mathfrak{b}^{\prime \prime}$ be the annihilator of the $\mathfrak{b}$-module $\mathfrak{d} / \mathfrak{c}$. Then $\mathfrak{b} / \mathfrak{b}^{\prime \prime}$ has dimension 1 or is the noncommutative Lie algebra of dimension 2 . So $\operatorname{Ker} \beta$ is an algebraic subalgebra.

Move (3.a). Assume that $\beta(\mathfrak{a})=0$ and $\mathfrak{t} \subset \mathfrak{a}$. Then set $\mathfrak{b}^{\prime}=\operatorname{Ker} \beta$ and $\mathfrak{a}^{\prime}=\mathfrak{a}$. We have $\mathfrak{b}^{\prime}(\lambda)=\mathfrak{b}(\lambda)+\mathfrak{t}$. Hence $\mathfrak{b}^{\prime}$ is a Pukhansky subalgebra. 
Move (3.b). Assume that $\beta(\mathfrak{a})=0$ and $\mathfrak{t} \not \subset \mathfrak{a}$. Then set $\mathfrak{b}^{\prime}=\mathfrak{b}$ and $\mathfrak{a}^{\prime}=\mathfrak{a}+\mathfrak{t}$. Move (3.c). Assume that $\beta(\mathfrak{a}) \neq 0$. Then set $\mathfrak{b}^{\prime}=\operatorname{Ker} \beta$ and $\mathfrak{a}^{\prime}=\mathfrak{a} \cap \mathfrak{b}^{\prime}+\mathfrak{t}$. We have $\mathfrak{b}^{\prime}(\lambda)=\mathfrak{b}(\lambda)+\mathfrak{t}$. Hence $\mathfrak{b}^{\prime}$ is a Pukhansky subalgebra.

The previous moves $(\mathfrak{a}, \mathfrak{b}) \mapsto\left(\mathfrak{a}^{\prime}, \mathfrak{b}^{\prime}\right)$ are called the admissible moves. Clearly $\left(\mathfrak{a}^{\prime}, \mathfrak{b}^{\prime}\right)$ belongs to $\mathscr{X}(\lambda)$ and we have $\operatorname{dim} \mathfrak{b}^{\prime} / \mathfrak{a}^{\prime}=\operatorname{dim} \mathfrak{b} / \mathfrak{a}-1$. Actually these moves were already used to prove the continuity of $I$ [CD, CV].

Lemma 2.6. Let $(\mathfrak{a}, \mathfrak{b}) \in \mathscr{X}(\lambda)$ with $\mathfrak{a} \neq \mathfrak{b}$. Then at least one of the conditions (2.5.1), (2.5.2), or (2.5.3) hold.

Proof. Assume that conditions (2.5.1) and (2.5.2) do not hold. We have $\mathfrak{b}(\lambda) \neq$ $\mathfrak{b}$ and $[\mathfrak{b}, \mathfrak{b}]=[\mathfrak{b}, u(\mathfrak{b})]$. Thus the $\mathfrak{b}$-module $\mathfrak{u}(\mathfrak{b}) / \mathfrak{c}$ is not trivial. Moreover, $\mathfrak{u}(\mathfrak{b}) / \mathfrak{c}$ contains a unique one-dimensional $\mathfrak{b}$-module $\mathfrak{z} / \mathfrak{c}$, and the $\mathfrak{b}$-module $\mathfrak{z} / \mathfrak{c}$ is trivial and contained in $\mathfrak{a} / \mathfrak{c}$. Then choose any two-dimensional $\mathfrak{b}$-module $\mathfrak{t} / \mathfrak{c}$ in $\mathfrak{u}(\mathfrak{b}) / \mathfrak{c}$. Then the assertion $(2.5 .3)$ follows.

Let $K(\lambda)$ be the free abelian group generated by $X(\mathfrak{g}(\lambda))$. We identify $K(\lambda)$ with the Grothendieck group of the category of finite-dimensional $\mathfrak{g}(\lambda)$-modules. For a $\mathfrak{g}(\lambda)$-module $M$ we denote by $[M]$ its image in $K(\lambda)$. Set $[M]=$ $\sum_{\alpha} m_{\alpha} e^{\alpha}$.

Let $a$ be an isotropic $\mathfrak{g}(\lambda)$-submodule of $\mathfrak{g}$ and let $a^{\prime}$ be its orthogonal relative to $B(\lambda)$. Set $\left[a^{\prime}\right]-[a]=\sum_{\alpha} m_{\alpha} e^{\alpha}$. Then the defect of $a$ is by definition the set $\operatorname{def}(a)=\left\{\alpha\right.$ such that $\left.m_{\alpha}+m_{-\alpha} \neq 0\right\}$. Let $\bar{Q}_{\lambda}(a)$ be the subgroup $\mathfrak{g}(\lambda)^{*}$ generated by the defect of $a$. Note that $\bar{Q}_{\lambda}(a) \subset Q(\mathfrak{g}(\lambda))$. Let $Q_{\lambda}(a)$ be the inverse image of $\bar{Q}_{\lambda}(a)$ in $Q(\mathfrak{g})$.

Let $\Sigma(a, \lambda)$ be the closure in (Grass $\mathfrak{g}) \times \mathfrak{g}^{*}$ of $G \cdot\left(a, \lambda+a^{\perp}\right)$. Set $X(a, \lambda)=$ $p_{2}(\Sigma(a, \lambda))$. As $p_{2}$ is a projective morphism, $X(a, \lambda)$ is exactly the closure in $\mathfrak{g}^{*}$ of $G \cdot\left(\lambda+a^{\perp}\right)$. For a Lie subalgebra $\mathfrak{a}$ of $\mathfrak{g}$ set $I(\mathfrak{a})=\mathfrak{a}^{\perp} \cap Q(\mathfrak{g})$. For an algebraic subalgebra $\mathfrak{a}$ such that $[\mathfrak{g}(\lambda), \mathfrak{a}] \subset \mathfrak{a}$ set $\Delta_{\lambda}(\mathfrak{a})=Q_{\lambda}(\mathfrak{a})+I(\mathfrak{a})$.

Lemma 2.7. Let $\mathfrak{a}$ be an isotropic algebraic subalgebra such that $[\mathfrak{g}(\lambda), \mathfrak{a}] \subset \mathfrak{a}$. Then we have $\lambda+k \otimes \Delta_{\lambda}(\mathfrak{a}) \subset X(\mathfrak{a}, \lambda)$.

Proof. (1) When $\mathfrak{a}$ is a Pukhansky polarization at $\lambda$, then the defect of $\mathfrak{a}$ is empty and $k \otimes \Delta_{\lambda}(\mathfrak{a})$ is orthogonal to $\mathfrak{a}$. In this case the lemma follows from the definitions.

(2) Set $\mathfrak{a}^{\prime}=\mathfrak{g}(\lambda)+\mathfrak{a}$. The elements of $\operatorname{def}(\mathfrak{a}) \backslash \operatorname{def}\left(\mathfrak{a}^{\prime}\right)$ are orthogonal to $\mathfrak{a} \cap \mathfrak{g}(\lambda)$. Hence we have $Q_{\lambda}(\mathfrak{a}) \subset I(\mathfrak{a})+Q_{\lambda}\left(\mathfrak{a}^{\prime}\right)$. Moreover, $X(\mathfrak{a}, \lambda)$ is stable by translation by $k \otimes I(\mathfrak{a})$. So it suffices to prove the lemma for $\mathfrak{a}^{\prime}$.

(3) By Lemma 2.6 there exists an integer $n$ and for $0 \leq i \leq n$ some elements $\left(\mathfrak{a}_{i}, \mathfrak{b}_{i}\right)$ of $\mathscr{X}(\lambda)$ such that

(2.7.1) $\mathfrak{a}_{0}=\mathfrak{a}$ and $\mathfrak{b}_{0}=\mathfrak{g}$.

(2.7.2) $\mathfrak{a}_{n}=\mathfrak{b}_{n}$; in particular, $\mathfrak{a}_{n}$ is a Pukhanky's polarization of $\lambda$.

(2.7.3) For $i<n,\left(\mathfrak{a}_{i}, \mathfrak{b}_{i}\right) \mapsto\left(\mathfrak{a}_{i+1}, \mathfrak{b}_{i+1}\right)$ is an admissible move.

Hence it suffices to prove the following assertion:

(2.7.4) If $(\mathfrak{a}, \mathfrak{b}) \mapsto\left(\mathfrak{a}^{\prime}, \mathfrak{b}^{\prime}\right)$ is an admissible move in $\mathscr{X}(\lambda)$, the assertion of 
Lemma 2.7 is true for $\mathfrak{a}$ whenever it is true for $\mathfrak{a}^{\prime}$.

Say that an admissible move $(\mathfrak{a}, \mathfrak{b}) \mapsto\left(\mathfrak{a}^{\prime}, \mathfrak{b}^{\prime}\right)$ is ineffective if $\mathfrak{a}=\mathfrak{a}^{\prime}$. For an ineffective move (i.e., a move of type (1.a) or (3.a)) assertion (2.7.4) is obvious.

(4) Let $(\mathfrak{a}, \mathfrak{b}) \mapsto\left(\mathfrak{a}^{\prime}, \mathfrak{b}^{\prime}\right)$ be an admissible move of type (3.b) with $\alpha(\mathfrak{a})=0$ or of type (1.b), (2). Then $\mathfrak{a}$ is an ideal of $\mathfrak{a}^{\prime}$ and we have $I(\mathfrak{a})=I\left(\mathfrak{a}^{\prime}\right)$. Thus we get $\Delta_{\lambda}(\mathfrak{a})=\Delta_{\lambda}\left(\mathfrak{a}^{\prime}\right)$. As we have $X\left(\mathfrak{a}^{\prime}, \lambda\right) \subset X(\mathfrak{a}, \lambda)$, assertion (2.7.4) follows.

(5) Let $(\mathfrak{a}, \mathfrak{b}) \mapsto\left(\mathfrak{a}^{\prime}, \mathfrak{b}^{\prime}\right)$ be an admissible move of type (3.b) with $\alpha(\mathfrak{a}) \neq 0$, or of type (1.c), (1.d). Choose $h \in \mathfrak{a}$ with $\alpha(h)=1$. When the move is of type (1.c) or (1.d) choose an element $y \in \mathfrak{d}-\mathfrak{c}$. Set $V=\{\exp t y, t \in k\}$. Set $\mathfrak{a}^{\prime \prime}=\operatorname{Ker} \alpha \mid \mathfrak{a}$ and set $X=\lambda+\mathfrak{a}^{\perp}, Y=\lambda+\mathfrak{a}^{\prime \prime}{ }^{\perp}$. We have $\left[y, \mathfrak{a}^{\prime \prime}\right] \subset \mathfrak{c}$. So for any $v \in V$ the restrictions of $v \cdot \lambda$ and $\lambda$ to $\mathfrak{a}^{\prime \prime}$ are the same. Hence $Y$ is $V$-stable. Moreover, for $\nu \in X$ we have $\nu((\exp \cdot t y) \cdot h)=\nu(h)-t \nu(y)$. Hence when $\nu(y)$ is not zero, $V \cdot \nu$ is not contained in $X$. As $X$ is a subvariety of codimension one in $Y$, we have $Y=\bar{V} \cdot X$. Hence $X(\mathfrak{a}, \lambda)$ contains $Y$. As $X\left(\mathfrak{a}^{\prime \prime}, \lambda\right)$ is the closure of $G \cdot Y$, we have $X(\mathfrak{a}, \lambda)=X\left(\mathfrak{a}^{\prime \prime}, \lambda\right)$. In particular, $X(\mathfrak{a}, \lambda)$ is stable by translation by $k \cdot \alpha$ and it contains $X\left(\mathfrak{a}^{\prime}, \lambda\right)$.

Moreover, $\Delta_{\lambda}(\mathfrak{a})=\Delta_{\lambda}\left(\mathfrak{a}^{\prime}\right)+\mathbf{F}_{p} \cdot \alpha$. Thus assertion (2.7.4) is proved in this case.

(6) Let $(\mathfrak{a}, \mathfrak{b}) \mapsto\left(\mathfrak{a}^{\prime}, \mathfrak{b}^{\prime}\right)$ be an admissible move of type (3.c) with $\alpha(\mathfrak{a})=0$. Choose $u \in \mathfrak{a}$ with $\beta(u)=1$. Set $V=\{\exp t y, t \in k\}, \mathfrak{a}^{\prime \prime}=\operatorname{Ker} \beta \mid \mathfrak{a}$, $X=\lambda+\mathfrak{a}^{\perp}$, and $Y=\lambda+\mathfrak{a}^{\prime \prime}{ }^{\perp}$. We have $\left[u, \mathfrak{a}^{\prime \prime}\right] \subset \mathfrak{c}$. So for any $v \in V$ the restrictions of $v \cdot \lambda$ and $\lambda$ to $\mathfrak{a}^{\prime \prime}$ are the same. Hence $Y$ is $V$-stable. Moreover, we have $\lambda((\exp \cdot t y) \cdot u)=\lambda(u)-t$. Hence $V \cdot \lambda$ is not contained in $X$. As $X$ is a subvariety of codimension one in $Y$, we have $Y=\overline{V \cdot X}$. Hence $X(\mathfrak{a}, \lambda)$ contains $Y$. As $X\left(\mathfrak{a}^{\prime \prime}, \lambda\right)$ is the closure of $G \cdot Y$, we have $X(\mathfrak{a}, \lambda)=X\left(\mathfrak{a}^{\prime \prime}, \lambda\right)$. In particular, $X(\mathfrak{a}, \lambda)$ contains $X\left(\mathfrak{a}^{\prime}, \lambda\right)$.

Moreover, $\Delta_{\lambda}(\mathfrak{a})=\Delta_{\lambda}\left(\mathfrak{a}^{\prime}\right)$. Thus assertion (2.7.4) is proved in this case.

(7) Let $(\mathfrak{a}, \mathfrak{b}) \mapsto\left(\mathfrak{a}^{\prime}, \mathfrak{b}^{\prime}\right)$ be an admissible move of type (3.c) with $\alpha(\mathfrak{a}) \neq 0$. Set $V=\{\exp t y, t \in k\}$. First we want to prove the following assertion.

(2.7.5) $X\left(\mathfrak{a}^{\prime}, \lambda\right)$ is contained in $X(\mathfrak{a}, \lambda)$.

Set $\mathfrak{a}^{\prime \prime}=\operatorname{Ker} \beta|\mathfrak{a} \cap \operatorname{Ker} \alpha| \mathfrak{a}$ and $\mathfrak{k}=\mathfrak{a}+\mathfrak{t}$. So we have $\mathfrak{a}^{\prime \prime}=\{a \in \mathfrak{a} \mid[a, \mathfrak{t}] \subset \mathfrak{c}\}$ and $\mathfrak{a}^{\prime \prime}$ is an ideal of $\mathfrak{k}$. Choose $h, x \in \mathfrak{a}$ such that $\alpha(h)=1, \alpha(x)=0, \beta(h)=$ 0 , and $\beta(x)=1$. Set $X=\lambda+\mathfrak{a}^{\perp}$ and $Y=\overline{V \cdot X}$. Thus $Y$ is a subvariety of $\lambda+\mathfrak{a}^{\prime \prime}{ }^{\perp}$. For $\nu \in X$ set $\nu_{t}=(\exp t y) \cdot \nu-\lambda$. We have

$$
\nu_{t}(h)=t \cdot \nu(y), \quad \nu_{t}(y)=\nu(y), \quad \nu_{t}(x)=t .
$$

Clearly $X \neq Y$. As $X$ is a subvariety of codimension 2 in $\lambda+\mathfrak{a}^{\prime \prime}, Y$ has codimension 1 and $Y$ is exactly the subset of all $\nu \in \lambda+\mathfrak{a}^{\prime \prime}$ which satisfy

$$
(\nu-\lambda)(h)=(\nu-\lambda)(x) \cdot(\nu-\lambda)(y) .
$$

We have $\mathfrak{a}^{\prime}=\mathfrak{a}^{\prime \prime} \oplus k \cdot h \oplus k \cdot y$. Hence we have $\lambda+\mathfrak{a}^{\prime \perp} \subset Y$ and assertion (2.7.5) follows. 
We have $[\mathfrak{a}]-\left[\mathfrak{a}^{\prime}\right]=e^{-\alpha^{\prime}}-e^{\alpha^{\prime}}$, where $\alpha^{\prime}$ is the restriction of $\alpha$ to $\mathfrak{g}(\lambda)$. Hence the defects of $\mathfrak{a}$ and $\mathfrak{a}^{\prime}$ are the same. Hence assertion (2.7.4) follows from (2.7.5).

\section{SemicontinUity PRINCIPLE}

The following lemma (which is adapted from [Q]) is a weak form of a "generic flatness" statement.

Lemma 3.1 (Quillen). Let $\mathbf{k}$ be a commutative noetherian ring without zero divisors, let $A$ be a finitely generated commutative $\mathbf{k}$-algebra, and let $\mathbf{g}$ be a $\mathbf{k}$-Lie algebra. Assume that $\mathbf{g}$ is a free $\mathbf{k}$-module of finite rank. Let $M$ be a cyclic $(A \otimes U(\mathbf{g}))$-module with a given generator $v$. Set $M^{\prime}=U(\mathbf{g}) \cdot v$. Then there exists a nonzero element $f \in \mathbf{k}$ such that $\mathbf{k}_{f} \otimes M^{\prime}$ and $\mathbf{k}_{f} \otimes\left(M / M^{\prime}\right)$ are torsion-free as $\mathbf{k}_{f}$-modules.

Proof. (1) As the set of torsion elements of $M^{\prime}$ is a $U(\mathbf{g})$-module of finite type, the assertion involving $M^{\prime}$ is clear. Now prove the assertion involving $M / M^{\prime}$.

(2) First assume that the k-algebra $A$ is generated by one element $x$. For any $n \geq 0$ set $M(n)=\sum_{0 \leq i \leq n} U(\mathbf{g}) x^{i} \cdot v$ and $X(n)=M(n) / M(n-1)$. Let $Y(n)$ be the submodule of k-torsion elements in $X(n)$. As $Y(n)$ is finitely generated as a $U(\mathbf{g})$-module, there is some nonzero element $g_{n}$ in $\mathbf{k}$ such that $g_{n} \cdot Y(n)=0$. Moreover, the multiplication by $x$ induces surjective morphisms of the $U(\mathbf{g})$-module $X(n) \rightarrow X(n+1)$. Hence there is some $N>0$ such that $X(n) \simeq X(N)$ for any $n \geq N$. Set $f=g_{1} \cdots g_{N}$. Then it is clear that $\mathbf{k}_{f} \otimes\left(M / M^{\prime}\right)$ is torsion-free.

(3) Now prove the lemma in general. Let $x_{1}, \ldots, x_{n}$ be a system of generators of the k-algebra $A$. For any integer $i$ with $1 \leq i \leq n$ set $\mathbf{g}_{i}=$ $\sum_{1 \leq j<i} \mathbf{k} x_{i} \oplus \mathbf{g}, A_{i}=\mathbf{k}\left[x_{i}\right], M_{i}^{\prime}=U\left(\mathbf{g}_{i}\right) \cdot v$, and $M_{i}=A_{i} \cdot M_{i}^{\prime}$. Thus, by point (1), we can find nonzero elements $f_{i} \in \mathbf{k}$ such that $\mathbf{k}_{f_{i}} \otimes\left(M_{i} / M_{i}^{\prime}\right)$ is torsionfree. Set $f=f_{1} \cdots f_{n}$. We have $M^{\prime}=M_{1}^{\prime}, M_{1}=M_{2}^{\prime}, \ldots, M_{n-1}=M_{n}^{\prime}$, and $M_{n}=M$. Hence the k-module $M / M^{\prime}$ has a finite filtration whose subquotients are $M_{i} / M_{i}^{\prime}$. Thus $\mathbf{k}_{f} \otimes M / M^{\prime}$ is torsion-free. Q.E.D.

Let $k$ be an algebraically closed field, let $(\mathfrak{g}, G)$ be a pair such that either

(3.1.1) $(\mathfrak{g}, G)$ is an algebraic solvable Lie algebra as defined in 1.5 and 1.7, or

(3.1.2) $k$ has characteristic zero, $\mathfrak{g}$ is a solvable Lie algebra, and $G$ is its algebraic adjoint group.

When (3.1.1) holds let $\mathscr{F}$ be a flag of $\mathfrak{g}$ as in 1.8. When (3.1.2) holds let $\mathscr{F}$ be any flag of ideals of $\mathfrak{g}$.

Let $X$ be a $G$-invariant irreducible subvariety of $\mathfrak{g}^{*}$. Let $K=k(X)$ be the field of rational functions over $X$. By scalar extension $\mathfrak{g}_{K}=\mathfrak{g} \otimes K$ is naturally a Lie algebra over $K$. As $\mathfrak{g}$ is a linear subspace of $S \mathfrak{g}$, the ring morphims $S \mathfrak{g} \rightarrow K$ gives rise to a $k$-linear map $\mathfrak{g} \rightarrow K$. Let $\lambda_{K}: \mathfrak{g}_{K} \rightarrow K$ be the corresponding $K$-linear form. Note that $K \otimes \mathscr{F}$ is a flag of ideals of $\mathfrak{g}_{K}$. 
Let $\mathfrak{p}(K)$ be the corresponding Vergne polarization associated with $\lambda_{K}$. The Borho-Gabriel-Rentschler module [BGR] is the $\mathfrak{g}_{K}$-module

$$
M\left(\lambda_{K}\right)=\operatorname{ind}^{*}\left(\mathfrak{p}(K), \mathfrak{g}_{K}\right) \lambda_{K} .
$$

Let $J(K)$ be the left ideal of $U\left(\mathfrak{g}_{K}\right)$ generated by elements $x-\lambda_{K}^{*}(x)$, $x \in \mathfrak{p}(K)$. Thus $J(K)$ is the left annihilator of the canonical generator of $M\left(\lambda_{K}\right)$. Consider $U(\mathfrak{g})$ as a subring of $U\left(\mathfrak{g}_{K}\right)$ and define $I(X)=J(K) \cap U(\mathfrak{g})$. As $I(X)$ is $G$-invariant, $I(X)$ is a two-sided ideal of $U(\mathfrak{g})$.

When $k$ has characteristic zero, $I(X)$ is prime and the corresponding map $I$ from the set $\operatorname{Spec}^{G} S \mathfrak{g}$ of $G$-invariant irreducible subvarieties of $\mathfrak{g}^{*}$ to the set $\operatorname{Spec}^{G} U(\mathfrak{g})$ of $G$-invariant prime ideals of $U(\mathfrak{g})$ does not depend on the choice of $\mathscr{F}$. The map $I$ is called the Dixmier map. It has been proved that $I$ is continous and bijective [BGR] (when $k$ has characteristic 0 any ideal of $U(\mathfrak{g})$ is $G$-invariant). Actually the proofs of these statements do not use the characteristic zero hypothesis. Hence these statements are also true in finite characteristic. However it will be easy to prove directly these statements in finite characteristic, so there is no need to assume them in characteristic $p$.

For simplicity we will now assume that case (3.1.1) holds, i.e., $(\mathfrak{g}, G)$ is an algebraic solvable Lie algebra over an algebraically closed field $k$ of characteristic 0 or $p \neq 2$.

It is clear that there exists a $G$-invariant affine, open, and smooth subset $X^{0}$ in $X$ on which the map $\lambda \in X^{0} \rightarrow \mathfrak{p}(\lambda)$ is a regular morphism from $X^{0}$ to the Grassmannian of $\mathfrak{g}$ (actually the following construction does not require $X^{0}$ to be $G$-invariant). Let $P X^{0}$ be the vector bundle over $X^{0}$ whose fiber over any point $\lambda \in X^{0}$ is $\mathfrak{p}(\lambda)$. It is a subbundle of the trivial bundle $\mathfrak{g} \times X^{0}$. Let $Q X^{0}$ be its quotient. So we have the following vector bundle exact sequence:

$$
0 \rightarrow P X^{0} \rightarrow \mathfrak{g} \times X^{0} \rightarrow Q X^{0} \rightarrow 0 .
$$

Set $\mathfrak{g}_{X^{0}}=\mathfrak{g} \otimes k\left[X^{0}\right]$ and let $\mathfrak{p}\left(X^{0}\right)$ be the global sections of $P X^{0}$. We define a $k\left[X^{0}\right]$-linear form $\lambda_{X^{0}}: \mathfrak{g}_{X^{0}} \rightarrow k\left[X^{0}\right]$ as previously. Note that $\mathfrak{g}_{X^{0}}, \mathfrak{p}\left(X^{0}\right)$, and $\lambda_{X^{0}}$ are $k\left[X^{0}\right]$-forms of $\mathfrak{g}_{K}, \mathfrak{p}(K)$, and $\lambda_{K}$. By construction, $\mathfrak{p}\left(X^{0}\right)$ is a subalgebra of $\mathfrak{g}_{X^{0}}$, the $k\left[X^{0}\right]$-module $\mathfrak{g}_{X^{0}} / \mathfrak{p}\left(X^{0}\right)$ is projective, and we have $\lambda_{X^{0}}\left(\left[\mathfrak{p}\left(X^{0}\right), \mathfrak{p}\left(X^{0}\right)\right]\right)=0$. Thus we can define the following module:

$$
M\left(\lambda_{X^{0}}\right)=\operatorname{ind}^{*}\left(\mathfrak{p}\left(X^{0}\right), \mathfrak{g}_{X^{0}}\right) \lambda_{X^{0}},
$$

which is a $k\left[X^{0}\right]$-form of the Borho-Gabriel-Rentschler module. Let $J\left(X^{0}\right)$ be the left annihilator of the canonical generator of $M\left(\lambda_{X^{0}}\right)$. As $M\left(\lambda_{X^{0}}\right)$ is torsion-free as a $k\left[X^{0}\right]$-module, it is clear that $J\left(X^{0}\right) \cap U(\mathfrak{g})=I(X)$.

It is possible to "thicken" all the previous objects. More precisely, there exists a finitely generated subring $\mathbf{k}$ of $k$ and objects $\mathbf{G}, \mathbf{g}, \mathbf{F}, \mathbf{X}, \mathbf{X}^{0}, \mathbf{P X}^{0}$, and $\mathbf{Q X ^ { 0 }}$ such that

(3.1.4) $\mathbf{g}$ is a free Lie algebra over $\mathbf{k}$ and $k \otimes \mathbf{g}=\mathfrak{g}$. Moreover, $\mathbf{F}$ is a flag of ideals of $\mathbf{g}$, the subquotients of $\mathbf{F}$ are free $\mathbf{k}$-modules, and $k \otimes \mathbf{F}=\mathscr{F}$. 
(3.1.5) $\mathbf{G}$ is a smooth affine group over $\mathbf{k}$ with Lie algebra $\mathbf{g}$ such that Spec $k \times \mathbf{G}=G$ and for any prime ideal $m$ of $\mathbf{k}$ the pair $(\kappa(m) \otimes \mathbf{g}, \operatorname{Spec} \kappa(m) \times$ G) is a solvable algebraic Lie algebra (where $\kappa(m)$ is any algebraically closed field containing $\mathbf{k} / m$ ) .

(3.1.6) $\mathbf{X}$ is a $G$-invariant irreducible closed flat subschme of $\mathbf{g}^{*}$, Spec $k \times$ $\mathbf{X}=X$, and for any prime ideal $m$ of $\mathbf{k}, \operatorname{Spec} \kappa(m) \times \mathbf{X}$ is reduced and irreducible.

(3.1.7) $\mathbf{X}^{0}$ is an affine $\mathbf{G}$-invariant open subset of $\mathbf{X}$, Spec $k \times \mathbf{X}^{0}=X^{0}$, and for any prime ideal $m$ of $\mathbf{k}, \operatorname{Spec} \kappa(m) \times \mathbf{X}^{0}$ is not empty.

(3.1.8) $\mathbf{P X} \mathbf{X}^{0}$ and $\mathbf{Q} \mathbf{X}^{0}$ are $\mathbf{G}$-equivariant locally free bundles over $\mathbf{X}^{0}$. Moreover, there is an exact sequence

$$
0 \rightarrow \mathbf{P X}^{0} \rightarrow \mathbf{g} \times \mathbf{X}^{0} \rightarrow \mathbf{Q} \mathbf{X}^{0} \rightarrow 0
$$

and after tensoring by $k$ it gives the exact sequence (3.1.3). Moreover, $\mathbf{P X}^{0}$ is stable by Lie bracket.

(3.1.9) $\mathbf{k}$ is integrally closed and 2 is invertible in $\mathbf{k}$.

Note that the ring $\mathbf{k}$ depends on $X$. However to prove the theorem only two varieties $X$ and $Y$ need to be considered at the same time. Set $\mathbf{g}_{\mathbf{X}^{0}}=\mathbf{g} \otimes \mathbf{k}\left[\mathbf{X}^{0}\right]$ and let $\mathbf{p}_{\mathbf{X}^{0}}$ be the global sections of $\mathbf{P X} \mathbf{X}^{0}$. As previously we define the canonical form $\lambda_{\mathbf{X}^{0}}$ attached to $\mathbf{X}^{0}$. By hypothesis $\mathbf{p}_{\mathbf{X}^{0}}$ is a Lie subalgebra of $\mathbf{g}_{\mathbf{X}^{0}}$, the $\mathbf{k}\left[\mathbf{X}^{0}\right]$-module $\mathbf{g}_{X^{0}} / \mathbf{p}_{X^{0}}$ is projective, and $\lambda_{\mathbf{X}}^{0}\left(\left[\mathbf{p}_{\mathbf{X}^{0}}, \mathbf{p}_{\mathbf{X}^{0}}\right]\right)=0$. Thus define the module

$$
\mathbf{M}\left(\lambda_{\mathbf{x}^{0}}\right)=\operatorname{ind}^{*}\left(\mathbf{p}_{\mathbf{x}^{0}}, \mathbf{g}_{\mathbf{X}^{0}}\right) \lambda_{\mathbf{x}^{0}},
$$

which is a $\mathbf{k}\left[\mathbf{X}^{0}\right]$-form of the Borho-Gabriel-Rentschler module. Let $\mathbf{J}\left(\mathbf{X}^{0}\right)$ be the left annihilator of the canonical generator of $\mathbf{M}\left(\lambda_{\mathbf{X}^{0}}\right)$. Set $\mathbf{I}(\mathbf{X})=\mathbf{J}\left(\mathbf{X}^{0}\right) \cap$ $U(\mathbf{g})$.

Lemma 3.2. (3.2.1) We have $\mathbf{I}\left(\mathbf{X}^{0}\right)=I(X) \cap U(\mathbf{g})$.

(3.2.2) For almost all ideals $m$ of $\mathbf{k}$ of height one, we have $\kappa(m) \otimes \mathbf{I}(\mathbf{X})=$ $I\left(\operatorname{Spec} \kappa(m) \times \mathbf{X}^{0}\right)$.

Proof. The canonical filtration of $U\left(\mathbf{g}_{\mathbf{X}^{0}}\right)$ induces a filtration of the module $\mathbf{M}\left(\lambda_{\mathbf{x}^{0}}\right)$. The graded group associated to this filtration is canonically isomorphic to $\mathbf{k}\left[\mathbf{Q X ^ { 0 }}\right]$. Hence $\mathbf{M}\left(\lambda_{\mathbf{x}^{0}}\right)$ is flat over $\mathbf{k}$ and over $\mathbf{k}\left[\mathbf{X}^{0}\right]$. In particular, $\mathbf{M}\left(\boldsymbol{\lambda}_{\mathbf{X}^{0}}\right)$ is torsion-free and the first assertion follows.

Using the exact sequence

$$
0 \rightarrow \mathbf{J}\left(\mathbf{X}^{0}\right) \rightarrow U\left(\mathbf{g}_{\mathbf{X}^{0}}\right) \rightarrow \mathbf{M}\left(\lambda_{\mathbf{x}^{0}}\right) \rightarrow 0
$$

we deduce that for any prime ideal $m$ of $\mathbf{k}$ we have

$$
\kappa(m) \otimes \mathbf{J}\left(\mathbf{X}^{0}\right)=J\left(\operatorname{Spec} \kappa(m) \times \mathbf{X}^{0}\right) .
$$

Moreover, it is clear that we have $\kappa(m) \otimes U(\mathbf{g})=U(\kappa(m) \otimes \mathbf{g})$. Set $A=\mathbf{k}\left[\mathbf{X}^{0}\right]$, $M=\mathbf{M}\left(\lambda_{\mathbf{x}^{0}}\right)$, and $M^{\prime}=U(\mathbf{g}) \cdot v$, where $v$ is the canonical generator of $M$. By Lemma 3.1, the k-modules $M^{\prime}$ and $M / M^{\prime}$ are torsion-free on a dense open 
subset of Speck. In particular, $\kappa(m) \otimes \mathbf{I}(\mathbf{X}) \rightarrow U(\kappa(m) \otimes \mathbf{g})$ is injective for almost all prime ideals of height one. Consider the following exact sequence:

$$
0 \rightarrow \mathbf{J}\left(\mathbf{X}^{0}\right) \cap U(\mathbf{g}) \rightarrow \mathbf{J}\left(\mathbf{X}^{0}\right) \oplus U(\mathbf{g}) \rightarrow U\left(\mathbf{g}_{\mathbf{X}^{0}}\right) \rightarrow M / M^{\prime} \rightarrow 0 .
$$

Unless $M / M^{\prime}$, all terms of the exact sequence are torsion-free $\mathbf{k}$-modules. Thus, by Lemma 3.1, for almost all height one ideals $m$ the tensor product of (3.2.3) with $\kappa(m)$ is still exact. So we get $\kappa(m) \otimes \mathbf{I}(\mathbf{X})=J\left(\operatorname{Spec} \kappa(m) \times \mathbf{X}^{0}\right) \cap$ $\kappa(m) \otimes U(\mathbf{g})$ for almost all $m$. Q.E.D.

Lemma 3.3. To prove the Theorem stated in the Introduction (over a field $k$ of characteristic 0 and for any solvable Lie algebra $\mathfrak{g}$ ) it suffices to prove the following statement:

(3.3.1) Let $(\mathfrak{g}, G)$ be any algebraic solvable Lie algebra over an algebraically closed field $\kappa$ of finite characteristic, and let $X, Y$ be any $G$-invariant irreducible subvarieties of $\mathfrak{g}^{*}$ such that $I(Y)$ is contained in $I(X)$. Then we have $X \subset Y$.

Proof. (1) First we claim that to prove the Theorem it suffices to prove it for algebraic solvable Lie algebras over fields of characteristic zero (actually this claim was known by the experts). So assume the theorem is proved in this case.

Let $k$ be an algebraically closed field of characteristic zero, let $\mathfrak{g}$ be any solvable Lie algebra, and let $\mathscr{A}$ be its adjoint group. Consider the following assertion:

(3.3.2) For any irreducible $\mathscr{A}$-invariant subvarieties $X$ and $Y$ of $\mathfrak{g}^{*}$ with $I(Y) \subset I(X)$, we have $X \subset Y$.

By definition of Zariski and Jacobson topologies the assertion is equivalent to the continuity of $I^{-1}$. Let $X, Y$ be irreducible $\mathscr{A}$-invariant subvarieties of $\mathfrak{g}^{*}$ such that $I(Y) \subset I(X)$. Let $\left(\mathfrak{g}^{\prime}, G^{\prime}\right)$ be an algebraic solvable Lie algebra as in Lemma 1.3 and let $\pi: \mathfrak{g}^{\prime *} \rightarrow \mathfrak{g}^{*}$ be the natural map. It is clear that the left ideal $U\left(\mathfrak{g}^{\prime}\right) \cdot I(X)$ is actually a two-sided ideal. Moreover, we have $U\left(\mathfrak{g}^{\prime}\right) \cdot I(X)=$ $I\left(\pi^{-1}(X)\right)$. Similarly the left ideal $U\left(\mathfrak{g}^{\prime}\right) \cdot I(Y)$ is actually a two-sided ideal and we have $U\left(\mathfrak{g}^{\prime}\right) \cdot I(Y)=I\left(\pi^{-1}(Y)\right)$. So we have $I\left(\pi^{-1}(Y)\right) \subset I\left(\pi^{-1}(X)\right)$. By hypothesis this implies $\pi^{-1}(X) \subset \pi^{-1}(Y)$. So we have $X \subset Y$.

(2) Let $k$ be an algebraically closed field of characteristic zero, and let $(\mathfrak{g}, G)$ be an algebraic solvable Lie algebra. As previously the proof of the bicontinuity of $I$ reduces to the following statement:

(3.3.3) For any irreducible $G$-invariant subvarieties $X$ and $Y$ of $\mathfrak{g}^{*}$ with $I(Y) \subset I(X)$, we have $X \subset Y$.

Recall that for any finitely generated subring $\mathbf{k}$ of $k$, the set of height one prime ideals with finite residual characteristic is a dense subset of Speck. Thus (3.3.3) follows from (3.3.1) and Lemma 3.2. Q.E.D.

\section{THE ORBITAL CORRESPONDENCE}

Let $k$ be an algebraically closed field of finite characteristic and let $(\mathfrak{g}, G)$ be an algebraic solvable Lie algebra. First we would like to heuristically describe 
the orbital correspondence. Let $\lambda \in \mathfrak{g}^{*}$. We can associate to $\lambda$ a map $\bar{\lambda}$ from $F_{*} \mathfrak{g}$ to $k$ by the formula $\bar{\lambda}(x)=\lambda(x)^{p}-\lambda\left(x^{p}\right)$. Usually $\bar{\lambda}$ is not additive, i.e., $\bar{\lambda}$ is not an element of $\left(F_{*} \mathfrak{g}\right)^{*}$. However there is a canonical way to associate to any coadjoint orbit $\mathscr{O}$ an orbit $C \mathscr{O}$ of $\left(F_{*} \mathfrak{g}\right)^{*}$. The construction is as follows. For any $\lambda \in \mathfrak{g}^{*}$, we define a central character $\bar{C} \lambda$ of $\mathfrak{p}(\lambda)$ by the formula $\bar{C} \lambda(x)=\lambda(x)^{p}-\lambda\left(x^{(p)}\right)$ for any $x \in \mathfrak{p}(\lambda)$. Then pick any $\lambda \in \mathscr{O}$ and choose any central character $E$ extending the central character $\bar{C} \lambda$ of $\mathfrak{p}(\lambda)$. Set $C \mathscr{O}=G \cdot E$. As we will see later the orbit $G \cdot E$ does not depend on the choices made to define it. In term of representation of $U(\mathfrak{g})$ the interpretation is the following. By the Dixmier map the orbit $\mathscr{O}$ is attached to a prime ideal $I(\overline{\mathscr{O}})$ and $\overline{C \mathscr{O}}$ is the closed subset of $F_{*} \mathfrak{g}$ defined by the ideal $I(\overline{\mathscr{O}}) \cap O(\mathfrak{g})$ of the algebra $O(\mathfrak{g})$ (see 1.7 ) which is canonically isomorphic to $k\left[F_{*} \mathfrak{g}\right]$. This section is devoted to the precise definition of the orbital correspondence.

Let $\mathfrak{a}$ be a restricted subalgebra of $\mathfrak{g}$ and let $\lambda \in X(\mathfrak{a})$. Define $C \lambda$ by the formula $C \lambda(x)=\lambda(x)^{p}-\lambda\left(x^{(p)}\right)$ for any $x \in \mathfrak{a}$. It follows from the Jacobson identity that $C \lambda$ is $p$-additive (see $[\mathrm{Ca}, \mathrm{WK}])$. Moreover, we have $[\mathfrak{a}, \mathfrak{a}] \subset \mathfrak{u}(\mathfrak{a})$. By Lemma 1.6.1 we have $C \lambda(x)=-\lambda\left(x^{(p)}\right)=0$ for any $x \in[\mathfrak{a}, \mathfrak{a}]$. Hence $C \lambda$ is a central weight of $\mathfrak{a}$.

Lemma 4.1. Let $\mathfrak{a}$ be a restricted subalgebra of $\mathfrak{g}$ and let $\lambda \in X(\mathfrak{a})$. Then the diagram

$$
0 \rightarrow Q(\mathfrak{a}) \rightarrow X(\mathfrak{a}) \stackrel{C}{\rightarrow} X_{F}(\mathfrak{a}) \rightarrow 0
$$

is an exact sequence.

Proof. (1) Set $\mathfrak{h}=\mathfrak{a} / \mathfrak{u}(\mathfrak{a}), Y=\mathfrak{h}^{*}$, and $Y_{F}=\left(F_{*} \mathfrak{h}\right)^{*}$. We claim that $0 \rightarrow$ $Q(\mathfrak{a}) \rightarrow Y \stackrel{C}{\rightarrow} Y_{F} \rightarrow 0$ is an exact sequence. As $\mathfrak{u}(\mathfrak{a})$ is a restricted ideal, $\mathfrak{h}$ inherits a natural $p$-structure. Set $\mathfrak{h}_{0}=\left\{h \in \mathfrak{h} \mid \alpha(h) \in \mathbf{F}_{p}\right.$ for any $\left.\alpha \in Q(\mathfrak{g})\right\}$. It is clear that $\mathfrak{h}_{0}$ is an $\mathbf{F}_{p}$-form of $\mathfrak{h}$. The eigeinvalue of any $h \in \mathfrak{h}_{0}$ on any simple subquotient of $\mathfrak{g}$ belongs to the prime field. Thus we have $h^{(p)}=h$ for any $h \in \mathfrak{h}_{0}$.

Choose a basis $\left(h_{i}\right)_{1 \leq i \leq n}$ of $\mathfrak{h}_{0}$, where $n=\operatorname{dim} \mathfrak{h}_{0}$. This identifies $Q(\mathfrak{a})$ with $\mathbf{F}_{p}^{n}$, and $Y, Y_{F}$ with $k^{n}$. Then $C$ is the map sending any $n$-tuple $\left(\lambda_{i}\right)_{1 \leq i \leq n}$ to the $n$-tuple $\left(\lambda_{i}^{p}-\lambda_{i}\right)_{1 \leq i \leq n}$. Thus the claim is obvious.

(2) The lemma now follows from the following remark. Let $\lambda \in X(\mathfrak{a})$ (respectively $\left.E \in X_{F}(\mathfrak{a})\right)$. Then $\lambda^{p}$ belongs to $X_{F}(\mathfrak{a})$ (respectively $E^{1 / p}$ belongs to $X(\mathfrak{a}))$ and $\lambda^{p}-C \lambda$ belongs to $Y_{F}$ (respectively $E^{1 / p}-\left(C\left(E^{1 / p}\right)\right)^{1 / p}$ belongs to $Y$ ). Q.E.D.

Lemma 4.2. Let $A \subset B$ be noetherian commutative rings. Let $l$ be an integer, let $\lambda_{1}, \ldots, \lambda_{l}$ be $l$ elements of $B$, let $E_{1}, \cdots, E_{l}$ be $l$ elements of $A$, and let $\left(m_{i, j}\right)_{1 \leq i, j \leq n}$ be an $(l \times l)$-matrix with entries in $A$. Assume that $B$ is generated over $A$ by $\lambda_{1}, \ldots, \lambda_{l}$ and that we have $\lambda_{i}^{p}=E_{i}+\sum_{1 \leq j \leq n} m_{i, j} \cdot \lambda_{j}$. Then the extension $A \subset B$ is finite. 
Proof. For an $l$-tuple $\alpha=\left(\alpha_{1}, \ldots, \alpha_{l}\right)$ of nonnegative integers set $\lambda^{\alpha}=$ $\lambda_{1}^{\alpha_{1}} \cdots \lambda_{l}^{\alpha_{l}}$. We have $B=\sum_{\alpha} A \cdot \lambda^{\alpha}$, where $\alpha=\left(\alpha_{1}, \ldots, \alpha_{l}\right)$ runs over the set of $l$-tuples such that $0 \leq \alpha_{i} \leq p-1$ for any $i \quad(0 \leq i \leq l)$. Thus $B$ is finite over $A$.

Lemma 4.3. Let $\lambda \in \mathfrak{g}^{*}$, let $E$ be a central character of $\mathfrak{g}$, and let $\mathfrak{q}$ be a restricted subalgebra of $\mathfrak{g}$ which is isotropic for $B(\lambda)$ and $B(E)$. Assume $C(\lambda \mid \mathfrak{q})=E \mid \mathfrak{q}$.

(4.3.1) If $\mathfrak{q}$ is a Pukhansky polarization at $\lambda$ (respectively at $E$ ) then it is a Pukhansky polarization at $E$ (respectively at $\lambda$ ).

(4.3.2) If $\mathfrak{q}$ is the Vergne polarization at $\lambda$ (respectively at $E$ ) then it is the Vergne polarization at $E$ (respectively at $\lambda$ ).

Proof. For $x \in \mathfrak{q} \cap \mathfrak{u}$ we have $\lambda(x)=E^{1 / p}(x)$. Hence there exists a linear form $\mu$ such that $\mu(x)=\lambda(x)$ for any $x \in \mathfrak{q}$ and $\mu(x)=E^{1 / p}(x)$ for any $x \in \mathfrak{u}$. Set $\alpha=E^{1 / p}-\mu$. Note that $\alpha \in \mathfrak{u}^{\perp}$.

Now assume that $\mathfrak{q}$ is a Pukhansky polarization at $\lambda$ (respectively at $E$ ). By Lemma 2.2.2, there exists $u \in \mathbf{U}(\mathfrak{q})$ such that $\operatorname{Ad}(u)(\mu)=\lambda$ (respectively $\left.\operatorname{Ad}(u)\left((\lambda+\alpha)^{p}\right)=E\right)$. Thus, in any case $\mathfrak{g}(\lambda)$ and $\mathfrak{g}(E)$ are conjugated by $\mathbf{U}(\mathfrak{q})$. Hence $\mathfrak{q}$ is simultaneously a Pukhansky polarization at $\lambda$ and $E$. Moreover we have $\mathfrak{p}(E)=\operatorname{Ad}(u)(\mathfrak{p}(\lambda))$. Hence if $\mathfrak{q}$ is the Vergne polarization at $\lambda$ or at $E$, it is the Vergne polarization at both $\lambda$ and $E$. Q.E.D.

Let $d$ be an integer. Let $P_{d}^{*}$ (respectively $P_{d, F}^{*}$ ) be the set of pairs $(\mathfrak{a}, \lambda)$ (respectively $(\mathfrak{a}, E)$ ), where $\mathfrak{a}$ is a $d$-dimensional restricted subalgebra of $\mathfrak{g}$ and where $\lambda$ is a weight of $\mathfrak{a}$ (respectively, $E$ is a central weight of $\mathfrak{a})$. As the set of $d$-dimensional restricted subalgebras of $\mathfrak{g}$ is a closed subset of Grass $_{d} \mathfrak{g}, P_{d}^{*}$ and $P_{d, F}^{*}$ are varieties. Define a morphism $C: P_{d}^{*} \rightarrow P_{d, F}^{*}$ by the formula $C(\mathfrak{a}, \lambda)=(\mathfrak{a}, C \lambda)$. Note that $Q(\mathfrak{g})$ acts by translation on $P_{d}^{*}$ and $C: P_{d}^{*} \rightarrow P_{d, F}^{*}$ takes constant value on $Q(\mathfrak{g})$-orbits (see Lemma 4.1).

Lemma 4.4. The morphism $C: P_{d}^{*} \rightarrow P_{d, F}^{*}$ is finite and surjective. Moreover, the fiber over any point of $P_{d}^{*}$ is exactly one $Q(\mathfrak{g})$-orbit.

Proof. Let $\mathscr{X}$ be the subvariety of Grass $\mathfrak{g}_{d}$ of restricted subalgebras of dimension $d$ and let $\mathscr{P}$ be the vector bundle over $\mathscr{X}$ whose fiber over $\mathfrak{a} \in \mathscr{X}$ is $\mathfrak{a}^{*}$. We can cover $\mathscr{X}$ by affine open subsets $U$ such that the restriction of $\mathscr{P}$ to $U$ is trivializable. Let $U$ be such an open subset and let $x_{1}, \ldots, x_{d}$ be sections of $\mathscr{P}$ over $U$ which trivialize that bundle over $U$. The $p$-structure defines a map $x \mapsto x^{(p)}$ on sections of $\mathscr{P}$ (this map is not additive). Define a matrix $\left(m_{i, j}\right)_{i, j}$ with coefficient in $k[U]$ by the relation $x_{i}^{(p)}=\sum_{1 \leq j \leq d} m_{i, j} x_{j}$. Let $V$ and $V_{F}$ be the inverse image of $U$ in $P_{d}^{*}$ and $P_{d, F}^{*}$. Define regular functions $\lambda_{i} \in k[V]$ (respectively $E_{i} \in k\left[V_{F}\right]$ ) by the formula $\lambda_{i}((\mathfrak{a}, \lambda))=\lambda\left(x_{i}\right)$ for any $(\mathfrak{a}, \lambda) \in V \quad\left(\right.$ respectively $E_{i}((\mathfrak{a}, E))=E\left(x_{i}\right)$ for any $\left.(\mathfrak{a}, E) \in V_{F}\right)$. Thus $k[V]$ is generated by $k[U]$ and $\lambda_{1}, \ldots, \lambda_{d}, k\left[V_{F}\right]$ is generated by $k[U]$ and 
$E_{1}, \ldots, E_{d}$, and we have

$$
\lambda_{i}^{p}=E_{i}+\sum_{1 \leq j \leq d} m_{i, j} \lambda_{j} .
$$

Hence by Lemma 4.2, $C$ is finite over $V_{F}$. As $U$ is arbitrary, $C$ is finite. Moreover, the assertion on the fibers of $C$ follows from Lemma 4.1. Q.E.D.

Let $d$ be an integer. Set $Y_{\text {reg }}=\left\{\lambda \in \mathfrak{g}^{*}\right.$ such that $\left.\operatorname{ind}(\lambda)=d\right\}$ and $Y_{F \text {, reg }}=$ $\left\{E \in\left(F_{*} \mathfrak{g}\right)^{*}\right.$ such that ind $\left.(E)=d\right\}$. Let $Y$ (respectively $Y_{F}$ ) be the closure of $Y_{\text {reg }}$ in $\mathfrak{g}^{*}\left(\right.$ respectively in $\left.\left(F_{*} \mathfrak{g}\right)^{*}\right)$.

Lemma 4.5. We have $C(\Theta(Y)) \subset \Theta\left(Y_{F}\right)$. Moreover, the induced morphism $C: \Theta(Y) \rightarrow \Theta\left(Y_{F}\right)$ is finite and surjective. The fiber over any point of $\Theta\left(Y_{F}\right)$ is exactly one $Q(\mathfrak{g})$-orbit.

Proof. Let $\lambda \in \mathfrak{g}^{*}$ and $E \in\left(F_{*} \mathfrak{g}\right)^{*}$, and let $\mathfrak{a}$ be a restricted subalgebra of dimension $d$ such that $\lambda \mid \mathfrak{a} \in X(\mathfrak{a})$ and $C(\lambda \mid \mathfrak{a})=E \mid \mathfrak{a}$.

(1) If $(\mathfrak{a}, \lambda \mid \mathfrak{a})$ belongs to $\Theta\left(Y_{\text {reg }}^{0}\right)$, then we have $\mathfrak{a}=\mathfrak{p}(\lambda)$. Then by Lemma 4.3 we also have $\mathfrak{a}=\mathfrak{p}(E)$ and $(\mathfrak{a}, E \mid \mathfrak{a})$ belongs to $\Theta\left(Y_{F, \text { reg }}\right)$. Thus we have $C\left(Y_{\text {reg }}^{0}\right) \subset Y_{F, \text { reg }}$.

(2) Similarly if $(\mathfrak{a}, E \mid \mathfrak{a})$ belongs to $\Theta\left(Y_{F}^{0}\right.$, reg $)$, we have $\mathfrak{a}=\mathfrak{p}(\lambda)=\mathfrak{p}(E)$ and $(\mathfrak{a}, \lambda \mid \mathfrak{a})$ belongs to $\Theta\left(Y_{\text {reg }}\right)$. Thus we have $C^{-1} Y_{F, \text { reg }}^{0} \subset Y_{\text {reg }}$.

(3) Moreover, by Lemma 4.4, the map $C$ is finite and the fiber of a point is one $Q(\mathfrak{g})$-orbit. So points (1) and (2) imply the lemma. Q.E.D.

Consider the following diagram:

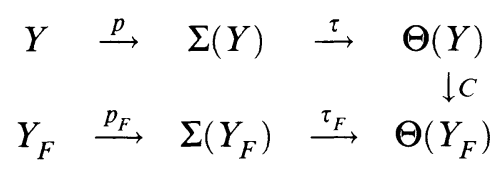

Let $X$ be a $G$-invariant locally closed subset of $Y$ of pure index $d$. Let $\Phi(X)$ (respectively $\Psi(X)$ ) be the set of triples $(\mathfrak{a}, \lambda, E)$ such that

(4.6.1) (a,$\lambda$ ) belongs to $\Sigma(X)$ (respectively to $\Theta(X)$ ) and $E$ to $\left(F_{*} \mathfrak{g}\right)^{*}$,

(4.6.2) $C(\lambda \mid \mathfrak{a})=E \mid \mathfrak{a}$ (respectively $C \lambda=E \mid \mathfrak{a})$.

Let $(\mathfrak{a}, \lambda, E)$ be an element of $\Phi(Y)$. By definition $(\mathfrak{a}, \lambda \mid \mathfrak{a})$ belongs to $\Theta(Y)$. Thus by Lemma 4.5, $(\mathfrak{a}, C(\lambda \mid \mathfrak{a}))$ is an element of $\Theta\left(Y_{F}\right)$. So by Lemma 2.2, $(\mathfrak{a}, E)$ is an element of $\Sigma\left(Y_{F}\right)$. Hence the map $p_{1,2} \times p_{1,3}$ realizes $\Phi(Y)$ as a closed subvariety of $\Sigma(Y) \times \Sigma\left(Y_{F}\right)$ : it is the subvariety of quadruples $(\mathfrak{a}, \lambda, \mathfrak{b}, E)$ of $\Sigma(Y) \times \Sigma\left(Y_{F}\right)$ such that $\mathfrak{a}=\mathfrak{b}$ and $C(\lambda \mid \mathfrak{a})=E \mid \mathfrak{a}$. Similarly $\Psi(Y)$ is naturally a closed subvariety of $\Theta(Y) \times \Sigma\left(Y_{F}\right)$.

It is clear that $\Phi(X)$ and $\Psi(X)$ are locally closed subvarieties of $\Phi(Y)$ and $\Psi(Y)$. The projection $p$ on the last factor sends $\Psi(Y)$ on $\left(F_{*} \mathfrak{g}\right)^{*}$. Set $C X=p(\Psi(X))$. With the notation of the previous diagram we have $C X=$ $p_{F} \circ \tau_{F}^{-1} \circ C \circ \tau \circ p^{-1}(X)$. 
Lemma 4.6. Let $X$ be a $G$-invariant irreducible subvariety of $\mathfrak{g}^{*}$. Then $\Psi(X)$ is irreducible, $C X$ is closed and irreducible, and the morphism $p: \Psi(X) \rightarrow C X$ is projective.

Proof. Let $d$ be the index of $X$. Let $\pi: \Phi(Y) \rightarrow \Sigma(Y)$ be the projection on the first two factors (where $Y$ is defined as in Lemma 4.5). Let $(\mathfrak{a}, \lambda)$ be an element of $\Sigma(Y)$. Then $\pi^{-1}(\mathfrak{a}, \lambda)$ is the set of triples $(\mathfrak{a}, \lambda, E)$, where $E$ is any central character extending $C(\lambda \mid \mathfrak{a})$. So the morphism $\pi$ is locally trivializable and has connected fibers. Moreover, we have $\Phi(X)=\pi^{-1}(\Sigma(X))$. Thus $\Phi(X)$ is closed and irreducible.

Let $\tau: \Phi(Y) \rightarrow \Psi(Y)$ be the natural projection. We have $\tau(\Phi(X))=\Psi(X)$. By Lemma 2.2, $\tau$ is locally trivializable and $\Phi(X)$ is $\tau$-saturated. So $\Psi(X)$ is a closed irreducible subset in $\Psi(Y)$. By Lemma 4.5, the natural morphism $\Psi(Y) \rightarrow \Sigma\left(Y_{F}\right)$ is finite. By Lemma 2.1, the map $\Sigma\left(Y_{F}\right) \rightarrow Y_{F}$ is projective. Hence the composed map $\Psi(Y) \rightarrow Y_{F}$ is projective. So $C X$ is an irreducible subvariety of $\left(F_{*} \mathfrak{g}\right)^{*}$ and $p: \Psi(X) \rightarrow C X$ is a projective morphism.

Section 4 contains the key to the proof. We will not use the next result in the proof of the main theorem. Its proof is given at the end of $\S 6$.

Theorem 4.7. Let $X$ be an irreducible G-invariant subvariety of $\mathfrak{g}^{*}$ and let $Y$ be an irreducible $G$-invariant subvariety of $\left(F_{*} \mathfrak{g}\right)^{*}$.

(4.7.1) $C X$ is an irreducible G-invariant subvariety of $\left(F_{*} \mathfrak{g}\right)^{*}$. It is defined by the ideal $I(X) \cap O(\mathfrak{g})$ of $O(\mathfrak{g}) \simeq S\left(F_{*} \mathfrak{g}\right)$.

(4.7.2) $C X$ is the set of central characters $E$ of $\mathfrak{g}$ such that $E \mid \mathfrak{p}=C(\lambda \mid \mathfrak{p})$ for some $\lambda \in X$ and some Pukhansky polarization $\mathfrak{p}$ at $\lambda$.

(4.7.3) We have $\operatorname{dim} C X=\operatorname{dim} X$.

(4.7.4) $C X$ is the closure of $a$ G-orbit if and only if $X$ is the closure of $a$ G-orbit.

(4.7.5) Let $C^{-1}(Y)$ be the set of irreducible $G$-invariant subvarieties $Z$ of $\mathfrak{g}^{*}$ such that $C Z=Y$. Then $C^{-1}(Y)$ contains exactly one $Q(\mathfrak{g})$-orbit.

\section{GOING UP LEMMAS}

Let $k$ be an algebraically closed field, let $A, B$ be finitely generated commutative $k$-algebras, let $\tau: A \rightarrow B$ be a morphism of $k$-algebras, let $E$ be an $A$-algebra, and let $F$ be a $B$-algebra. A morphism of $A$-algebras $\pi: E \rightarrow F$ is called an extension if the induced map $B \otimes_{A} E \rightarrow F$ is a surjective morphism of $B$-algebras. So for an extension $\pi: E \rightarrow F$ we have:

(5.0.1) $\pi(E)$ commutes with $B$, and

(5.0.2) $F=B \cdot \pi(E)$.

Actually an "extension" as defined here is usually called a "central extension" in the literature. Recall that any extension $\pi: E \rightarrow F$ induces a map $\pi^{-1}$ : $\operatorname{Spec} F \rightarrow \operatorname{Spec} E$ as in the commutative case [P].

Similarly let $X, Y$ be varieties over $k$, let $\tau: Y \rightarrow X$ be a morphism of varieties, let $\mathscr{E}$ be an $\mathscr{O}_{X}$-algebra, and let $\mathscr{F}$ be an $\mathscr{O}_{Y}$-algebra (see 1.9). A 
morphism of $\mathscr{O}_{X}$-algebras $\pi: \mathscr{E} \rightarrow \tau_{*} \mathscr{F}$ is called an extension if the induced map $\tau^{*} \mathscr{E} \rightarrow \mathscr{F}$ is a surjective morphism of $\mathscr{O}_{Y}$-algebras. Similarly any extension $\pi: \mathscr{E} \rightarrow \tau_{*} \mathscr{F}$ induces a map $\pi^{-1}: \operatorname{Spec} \mathscr{F} \rightarrow \operatorname{Spec} \mathscr{E}$.

Let $X$ be a variety and let $\mathscr{D}$ be an $\mathscr{O}_{X}$-algebra. Then for any prime ideal $\mathscr{P}$ of $\mathscr{D}$, the $\mathscr{O}_{X}$-ideal $m=\mathscr{P} \cap \mathscr{O}_{X}$ is a prime ideal of $\mathscr{O}_{X}$. By definition $m$ is the ideal of $\mathscr{O}_{X}$ associated with $\mathscr{P}$. Moreover, when $\mathscr{D}$ is coherent over $\mathscr{O}_{X}$ and $\mathscr{P}$ is maximal then $m$ is a maximal ideal. Thus $\mathscr{O}_{X} / m$ can be identified with $k$ and $\mathscr{D} / \mathscr{P}$ is a simple algebra of finite dimension over $k$.

Lemma 5.1. Let $X$ be an irreducible variety and let $\mathscr{D}$ be a prime coherent $\mathscr{O}_{X}$-algebra. Let $\mathscr{M}$ be a $\mathscr{D}$-module which is coherent and torsion-free as an $\hat{O}_{X}$-module. Let $\mathscr{R}$ be a maximal ideal of $\mathscr{D}$ and let $m$ be the corresponding ideal of $\mathscr{O}_{X}$. Then $\mathscr{R}$ is the annihilator of a simple subquotient of $\mathscr{M} / m \cdot \mathscr{M}$. Proof. It is clear that we could use an affine neighborhood of the point defined by $m$ instead of $X$. So we can assume that $X$ is affine. Let $O, D, M, R$, and $m$ be the global sections of sheaves $\mathscr{O}_{X}, \mathscr{D}, \mathscr{M}, \mathscr{R}$, and $m$. For any nonzero $x$ in $M$, let $I(x)=\{d \in D \mid d \cdot x=0\}$ be its left annihilator and let $\mathscr{I}$ be the collection of all $I(x)$. For $I \in \mathscr{I}$ set $M(I)=\{m \in M \mid I \cdot m=0\}$. Note that the subgroups $M(I)$ are $O$-modules and their union is $M$. Hence $M$ can be written as a finite sum of $M(I)$. So we can find an integer $n>0$ and $I_{1}, \ldots, I_{n} \in \mathscr{I}$ such that $I_{1} \cap \cdots \cap I_{n}=0$. Thus $M^{n}$ contains a submodule isomorphic to the left $D$-module $D$.

Let $\rho$ be the unique simple $D / R$-module. As it is a simple quotient of $M^{n}$, $\rho$ can be realized as a subquotient of $M$. Let $M^{\prime \prime} \subset M^{\prime}$ be submodules of $M$ such that $M^{\prime} / M^{\prime \prime}$ is isomorphic to $\rho$. For any $l \geq 0$ set $M(l)=m^{l} \cdot M$. As $M$ is torsion-free and coherent as an $O$-module, the $m$-adic topology of $M$ is separated. So there exists an integer $l>0$ such that we have $M^{\prime} \subset M(l)$ and $M^{\prime} \not \subset M(l+1)$. We have $m \cdot M^{\prime} \subset M^{\prime \prime}$. Thus $\rho$ can be realized as a subquotient of $M(l) / M(l+1)$. Let $x_{1}, \ldots, x_{j}$ be a set of generators of the ideal $m$. Let $\alpha=\left(\alpha_{1}, \ldots, \alpha_{j}\right)$ be a $j$-tuple of nonnegative integers with $\sum \alpha_{i}=l$. Then the multiplication by $x_{1}^{\alpha_{1}} \cdots x_{j}^{\alpha_{j}}$ induces a map $\tau_{\alpha}: M / m \cdot M \rightarrow M(l) / M(l+1)$. Using the maps $\tau_{\alpha}$, we can realize $M(l) / M(l+1)$ as a quotient of copies $M / m \cdot M$. Thus $\rho$ is a subquotient of $M / m \cdot M$.

Lemma 5.2. Let $X, Y$ be irreducible varieties and let $\tau: Y \rightarrow X$ be a projective and surjective morphism of varieties. Let $\mathscr{E}$ be a prime coherent torsion-free $\mathscr{O}_{X}$-algebra, let $\mathscr{F}$ be a torsion-free $\mathscr{O}_{Y}$-algebra, and let $\pi: \mathscr{E} \rightarrow \pi_{*} \mathscr{F}$ be an extension. Let $\mathscr{R}$ be a maximal ideal of $\mathscr{E}$. Then there exists a maximal ideal $\mathscr{M}$ of $\mathscr{F}$ such that $\pi^{-1} \mathscr{M}=\mathscr{R}$.

Proof. Set $m=\mathscr{R} \cap \mathscr{O}_{X}$, and let $x$ be the point of $X$ associated with $m$. It suffices to prove the lemma in a neighborhood of $x$. So we can assume that $X$ is affine. Let $\mathscr{L}$ be a $\tau$-ample line bundle over $Y$. Let $A, m, E, R, B_{n}, F_{n}$ be the global sections of $\mathscr{O}_{X}, m, \mathscr{E}, \mathscr{R}, \mathscr{L}^{\otimes n}, \mathscr{F} \otimes \mathscr{L}^{\otimes n}$, respectively, where $n$ is any nonnegative integer. Set $B=\bigoplus_{n \geq 0} B_{n}$ and $F=\bigoplus_{n \geq 0} F_{n}$. Set 
$\bar{E}=E / m E$ and $\bar{F}=F / m F$, and let $\bar{\pi}: \bar{E} \rightarrow \bar{F}$ be the morphism induced by $\pi$. Let $\bar{R}$ be the ideal of $\bar{E}$ induced by $R$. The map Spec $\tau_{*} \mathscr{O}_{Y} \rightarrow X$ is finite. Thus we have $F_{0}=B_{0} \cdot E$. Moreover, using a sufficiently large power of $\mathscr{L}$ instead of $\mathscr{L}$ we can always assume that for any $n \geq 0$ we have $B_{1} \cdot F_{n}=F_{n+1}$. Thus $E \subset F$ is an extension.

Look at $\bar{F}$ as a left $\bar{F}$-module. It has a finite filtration $\mathscr{G}$ by graded submodules such that the annihilator $p(C)$ of any suquotient $C$ of the filtration $\mathscr{G}$ is prime. Let $\operatorname{Sub} \mathscr{G}$ be the set of subquotients of $\mathscr{G}$. For any $C \in \operatorname{Sub} \mathscr{G}$ the ideal $m(C)=\bar{\pi}^{-1}(p(C))$ is a maximal ideal of $\bar{E}$ and the $\bar{E}$-module $C$ is isotypical (its type is the unique simple $(\bar{E} / m(C))$-module). By Lemma 5.1, the unique simple $(\bar{E} / \bar{R})$-module appears as a subquotient of each $F_{n} / m \cdot F_{n}$. Hence there exists at least one infinite-dimensional subquotient $C \in \operatorname{Sub} \mathscr{G}$ such that $m(C)=\bar{R}$. As $C$ is finitely generated as an $F$-module, the prime ideal $p(C)$ has infinite codimension in $\bar{F}$. Thus we have found a homogenous prime ideal $p$ of infinite codimension in $F$ such $p \cap E=R$. So $p$ defines an ideal $\mathscr{P}$ of $\mathscr{F}$ such that $\pi^{-1} \mathscr{P}=\mathscr{R}$. Thus any maximal ideal $\mathscr{M}$ of $\mathscr{F}$ containing $\mathscr{P}$ satisfies the assertion of the lemma.

\section{Proof of The Theorem}

Let $k$ be an algebraically closed field of characteristic $p$ and let $\mathfrak{k}$ be a restricted algebra. For any central character $E$ of $\mathfrak{k}$, let $m(E)$ be the corresponding maximal ideal of $O(\mathfrak{k})$. We say that a k-module $N$ admits $E$ as a central character if $m(E) \cdot N=0$. We set $U_{E}(\mathfrak{k})=U(\mathfrak{k}) / m(E) \cdot U(\mathfrak{k})$. Given a weight $\lambda$ of a restricted subalgebra $\mathfrak{k}^{\prime}$, we set

$$
\operatorname{ind}_{E}\left(\mathfrak{k}^{\prime}, \mathfrak{k}\right) \lambda=\operatorname{ind}\left(\mathfrak{k}^{\prime}, \mathfrak{k}\right) \lambda / m(E) \cdot \operatorname{ind}\left(\mathfrak{k}^{\prime}, \mathfrak{k}\right) \lambda .
$$

Note that $\operatorname{ind}_{E}\left(\mathfrak{k}^{\prime}, \mathfrak{k}\right) \lambda=0$ unless we have $C \lambda=E \mid \mathfrak{k}^{\prime}$.

As previously, let $\mathfrak{g}$ be an algebraic solvable Lie algebra. Let $\mathscr{T}$ be the variety of the triple $(\mathfrak{a}, \lambda, E)$, where $\mathfrak{a}$ is a restricted Lie subalgebra of $\mathfrak{g}, \lambda$ is a weight of $\mathfrak{a}$, and $E$ is a central character with $C \lambda=E \mid \mathfrak{a}$. Let $\mathscr{V}$ be the subset of $\mathscr{T}$ of the triple $(\lambda, \mathfrak{a}, E)$ such that $\mathfrak{a}$ is a polarization at $E$.

Let $(\mathfrak{a}, \lambda, E)$ be an element of $\mathscr{T}$. For any $g \in \mathfrak{a}$, set $\lambda^{*}(g)=\lambda(g)+$ $\frac{1}{2} \operatorname{Tr}(\operatorname{ad} g \mid \mathfrak{g} / \mathfrak{a})$. Set $M(\mathfrak{a}, \lambda, E)=\operatorname{ind}_{E}(\mathfrak{a}, \mathfrak{g}) \lambda^{*}$. Let $L(\mathfrak{a}, \lambda, E)$ be the annihilator of this module. For any integer $n$ let $S_{n}$ be the set of $n$-tuples $\left(\alpha_{1}, \ldots, \alpha_{n}\right)$, where the integers $\alpha_{i}$ satisfy $0 \leq \alpha_{i} \leq p-1$. Recall the following lemma (see $[\mathrm{SF}])$.

Lemma 6.1 (Poincaré, Birkhoff, Witt). Let $(\mathfrak{a}, \lambda, E)$ be in $\mathscr{T}$. Set $n=\operatorname{dim} \mathfrak{g}$ and $l=\operatorname{codim} \mathfrak{a}$.

(6.1.1) Let $x_{1}, \ldots, x_{n}$ be a basis of $\mathfrak{g}$. Then $x_{1}^{\alpha_{1}} \cdots x_{n}^{\alpha_{n}}$, when $\left(\alpha_{1}, \ldots, \alpha_{n}\right)$ runs over $S_{n}$, is a basis of $U_{E}(\mathfrak{g})$.

(6.1.2) Let $v$ be the canonical generator of $M(\mathfrak{a}, \lambda, E)$. Let $x_{1}, \ldots, x_{l}$ be a basis of a complement of $\mathfrak{a}$. Then $x_{1}^{\alpha_{1}} \cdots x_{l}^{\alpha_{l}} \cdot v$, when $\left(\alpha_{1}, \ldots, \alpha_{l}\right)$ runs over $S_{l}$, is a basis of $M(\mathfrak{a}, \lambda, E)$. 
Theorem 6.2 (Weisfeiler, $\mathrm{Kac}$ ). (6.2.1) Let $\mathfrak{k}$ be a restricted solvable algebra, let $E$ be a central character of $\mathfrak{k}$, and let $2 r$ be the rank of $B(E)$. Then any simple $\mathfrak{k}$-module with central character $E$ has dimension $p^{r}$.

(6.2.2) For any $(\mathfrak{a}, \lambda, E) \in \mathscr{V}$, the module $M(\mathfrak{a}, \lambda, E)$ is simple.

(6.2.3) Let $E$ be a central character of $\mathfrak{g}$, let $\mathfrak{p}$ be a polarization of $E$, and let $M$ be a simple $\mathfrak{g}$-module with central character $E$. Then $M$ is isomorphic to $M(\mathfrak{p}, \lambda, E)$ for some $\lambda \in X(\mathfrak{p})$ such that $C \lambda=E$.

Proof. For (6.2.1) see [WK]. Assertion (6.2.2) follows from (6.1.2) and (6.2.1). Let $M$ be as in (6.2.3). Any simple $\mathfrak{p}$-submodule of $M$ has dimension 1 . Hence $M$ is isomorphic to some $M(\mathfrak{p}, \lambda, E)$. Q.E.D.

Let $X$ be a variety. A $U(\mathfrak{g})$-vector bundle over $X$ is a vector bundle endowed with an action of $U(\mathfrak{g})$ which commutes with $\mathscr{O}_{X}$. Let $\phi: X \rightarrow \mathscr{T}$ be a morphism. By Lemma 6.1, there exists a natural vector bundle $\mathscr{M}$ over $X$ whose fiber over a point $x \in X$ is $M(\phi(x))$. Let $L(X)$ be the annhilator in $U(\mathfrak{g})$ of $\mathscr{M}$.

For any weight $\lambda$ of $\mathfrak{g}$, define an automorphism $\tau_{\lambda}$ of $U(\mathfrak{g})$ by the formula $\tau_{\lambda}(g)=g-\lambda(g)$ for any $g \in \mathfrak{g}$. In this way $X(\mathfrak{g})$ acts on $U(\mathfrak{g})$. We have

$$
\tau_{\lambda}(\sigma(g))=\sigma(g)+C \lambda(g)
$$

for any $g \in \mathfrak{g}$. Thus $\tau_{\lambda}$ stabilizes $O(\mathfrak{g})$ and acts on $O(\mathfrak{g})$ as the translation by $C \lambda$. By Lemma $4.1, Q(\mathfrak{g})$ acts trivially on $O(\mathfrak{g})$. Hence $Q(\mathfrak{g})$ acts naturally on the set of simple $\mathfrak{g}$-modules with a given central character $E$.

Lemma 6.3. Let $(\mathfrak{a}, \lambda, E) \in \mathscr{T}$.

(6.3.1) The closure $X$ in $\mathscr{T}$ of the $G(E)$-orbit of $(\mathfrak{a}, \lambda, E)$ contains $a G(E)$ invariant point. Moreover, for any element $\left(\mathfrak{a}^{\prime}, \lambda^{\prime}, E\right) \in X$ the simple subquotients of $M\left(\mathfrak{a}^{\prime}, \lambda^{\prime}, E\right)$ and $M(\mathfrak{a}, \lambda, E)$ are the same.

(6.3.2) Let $\mathfrak{b}$ be a restricted $B(E)$-isotropic subalgebra of $\mathfrak{g}$. Assume that we have $\mathfrak{b}=\mathfrak{u}(\mathfrak{b})+\mathfrak{c}$, where $\mathfrak{c}=\mathfrak{a} \cap \mathfrak{b}$. Then $M(\mathfrak{a}, \lambda, E)$ contains a nonzero vector $v^{\prime}$ such that $u \cdot v^{\prime}=E(u)^{1 / p} \cdot v^{\prime}$ for any $u \in \mathfrak{u}(\mathfrak{b})$ and $c \cdot v^{\prime}=\left(\lambda^{*}(c)-\right.$ $\operatorname{Tr}(\operatorname{ad} c \mid \mathfrak{b} / \mathfrak{c})) \cdot v^{\prime}$ for any $c \in \mathfrak{c}$.

(6.3.3) The set of simple subquotients of $M(\mathfrak{a}, \lambda, E)$ is stable under $I(\mathfrak{a})$.

(6.3.4) Assume that there exists $\left(\mathfrak{a}^{\prime}, \lambda^{\prime}, E\right)$ in $\mathscr{T}$ such that $\mathfrak{a}$ is an ideal of $\mathfrak{a}^{\prime}$ and $\lambda^{\prime} \mid \mathfrak{a}=\lambda$. Then any simple subquotient of $M(\mathfrak{a}, \lambda, E)$ is in the $I(\mathfrak{a})$-orbit of some simple subquotient of $M\left(\mathfrak{a}^{\prime}, \lambda^{\prime}, E\right)$.

Proof. (1) Set $\mathfrak{q}=\mathfrak{a}+\mathfrak{u}$. For any $x \in \mathfrak{u}(\mathfrak{a})$ we have $\lambda(x)^{p}=E(x)$. Hence there exists a unique element $\mu \in \mathfrak{q}^{*}$ such that $\lambda(x)=\mu(x)$ for any $x \in \mathfrak{a}$ and $\mu(x)^{p}=E(x)$ for any $x \in \mathfrak{u}$. As $[\mathfrak{g}(E), \mathfrak{g}] \subset \mathfrak{u}, \mu$ is $\mathfrak{g}(E)$-invariant. By Lemma 1.6.2, $\mu$ is $G(E)$-invariant. Let $Y$ be the closure of $G(E) \cdot \mathfrak{a}$ in Grass $\mathfrak{g}$. Thus $p_{1}: X \rightarrow Y$ is an isomorphism (its inverse is $\mathfrak{c} \mapsto(\mathfrak{c}, \mu \mid \mathfrak{c}, E)$ ). Hence $X$ is a projective variety and by Borel's Fixed Point Theorem $X$ contains a $G(E)$-invariant point. Moreover, by Lemma $6.1, U_{E}(\mathfrak{g})$ is a finitedimensional algebra and for any $x \in X, M(x)$ is the fiber of a natural 
$U_{E}$-vector bundle $\mathscr{M}$ over $X$. Hence the set of simple subquotients of $M(x)$ (and their multiplicities) is independant of $x$. This proves (6.3.1).

(2) Let $v$ be the canonical generator of $M(\mathfrak{a}, \lambda, E)$ and set $N=U(\mathfrak{b}) \cdot v$. Let $F$ and $F^{\prime}$ be the restrictions of $E$ to $\mathfrak{b}$ and $\mathfrak{u}(\mathfrak{b})$. By the PoincaréBirkhoff-Witt Theorem we have $N \simeq \operatorname{ind}_{F}(\mathfrak{c}, \mathfrak{b}) \lambda^{*} \mid \mathfrak{c}$ as a $\mathfrak{b}$-module and $N \simeq$ $\operatorname{ind}_{F^{\prime}}(\mathfrak{u}(\mathfrak{c}), \mathfrak{u}(\mathfrak{b})) \lambda^{*} \mid \mathfrak{u}(\mathfrak{c})$ as a $\mathfrak{u}(\mathfrak{b})$-module. Note that any simple subquotient of the $\mathfrak{b}$-module $N$ has dimension 1 .

Let $V$ be a vector space of dimension $n$. Let $\bar{S} V$ be the quotient of $S V$ by the ideal generated by all $x^{p}, x \in V$. Then $\bar{S} V$ is a graded algebra of dimension $p^{n}$. Let $\bar{V}$ be its component of degree $n(p-1)$, i.e., the component of highest degree of $\bar{S} V$. Then $\bar{V}$ has dimension 1 and we have $\bar{V}=\{y \in \bar{S} V$ such that $V \cdot y=0\}$. Moreover, there is a canonical isomorphism res : $\left(\bigwedge^{n} V\right)^{\otimes p-1} \rightarrow \bar{V}$. If $x_{1}, \ldots, x_{n}$ is a basis of $V$ then we have $\operatorname{res}\left(x_{1} \wedge \cdots \wedge x_{n}\right)=x_{1}^{p-1} \cdots x_{n}^{p-1} \quad\left(\right.$ actually this map is a residue map, see $\left.\left[\mathrm{M}_{1}\right]\right)$.

Now set $V=\mathfrak{u}(\mathfrak{b}) / \mathfrak{u}(\mathfrak{c})=\mathfrak{b} / \mathfrak{c}$. The canonical filtration of $U(\mathfrak{u}(\mathfrak{p}))$ induces a filtration $k \cdot v=N_{0} \subset N_{1} C \cdots$ of $N$ and the associated graded vector space is naturally isomorphic to $\bar{S} V$. For any $g \in \mathfrak{b}$, denote by $\rho(g)$ its action on $N$ and by $\bar{g}$ its image in $\mathfrak{b} / \mathfrak{c}$. We have $\sigma_{i+1}(\rho(g) n)=\bar{g} \cdot \sigma_{i}(n)$ for any $i \in \mathbb{Z}_{+}$, $n \in N_{i}, g \in \mathfrak{b}$, where $\sigma_{j}: N_{j} \rightarrow \bar{S} V$ is the symbol map. So $N_{(p-1) n-1}$ contains no nonzero $\mathfrak{u}(\mathfrak{b})$-submodule. Thus the simple components of the socle $S$ of the $\mathfrak{u}(\mathfrak{b})$-module $N$ have no multiplicities. As we have $\rho(u) \mid S=E(u)^{1 / p}$ for any $u \in \mathfrak{u}(\mathfrak{b}), S$ is isotypical. So we have $\operatorname{dim} S=1$.

In particular, $S$ is a b-module. Moreover, the natural map $S \rightarrow$ $N_{n(p-1)} / N_{n(p-1)-1} \otimes \lambda^{*} \mid \mathfrak{c}$ is an isomorphism of $\mathfrak{c}$-modules. Using the map res we get that any $g \in \mathfrak{c}$ acts on $\bar{V}$ as the scalar $(p-1) \operatorname{Tr}(\operatorname{ad}(g) \mid V)=-\operatorname{Tr}(\operatorname{ad} g \mid \mathfrak{b} / \mathfrak{c})$. Thus any $c \in \mathfrak{c}$ acts on $S$ as $\lambda^{*}(c)-\operatorname{Tr}(\operatorname{ad} c \mathfrak{b} / \mathfrak{c})$. So any nonzero vector $v^{\prime} \in S$ satisfies assertion (6.3.2).

(3) Assertion (6.3.3) is obvious because $M(\mathfrak{a}, \lambda, E)$ is stable under $I(\mathfrak{a})$.

(4) Let $v$ be the canonical generator $M(\mathfrak{a}, \lambda, E)$ and set $N=U\left(\mathfrak{a}^{\prime}\right) \cdot v$. Then all the simple subquotients of the $\mathfrak{a}^{\prime}$-module $N$ have dimension 1 and the corresponding weights are $\lambda^{\prime *}+\alpha \mid \mathfrak{a}^{\prime}$ for some $\alpha \in I(\mathfrak{a})$. Hence $M(\mathfrak{a}, \lambda, E)$ has a filtration whose subquotients are $M\left(\mathfrak{a}^{\prime}, \lambda^{\prime}+\alpha \mid \mathfrak{a}^{\prime}, E\right)$ for some $\alpha \in I(\mathfrak{a})$. Thus (6.3.4) follows from (6.3.3).

Lemma 6.4. Let $(\mathfrak{p}, \lambda, E)$ and $(\mathfrak{q}, \mu, E)$ be elements of $\mathscr{V}$. The two simple modules $M(\mathfrak{p}, \lambda, E)$ and $M(\mathfrak{q}, \mu, E)$ are isomorphic if and only if the restrictions of $\lambda$ and $\mu$ to $\mathfrak{g}(E)$ are the same.

Proof. (1) First assume that the restrictions are the same and prove that the modules are isomorphic. We can assume that $\mathfrak{q}$ is a Pukhansky polarization at $E$. Set $\mathfrak{c}=\mathfrak{p} \cap \mathfrak{q}$. Note that $\mathfrak{c}$ contains $\mathfrak{g}(E)$. So we have $\mathfrak{q}=\mathfrak{u}(\mathfrak{q})+\mathfrak{c}$.

For $x \in \mathfrak{g}(E)$, let $c(x)$ (respectively $p(x), p^{\prime}(x), q(x), q^{\prime}(x)$ ) be the trace of $\operatorname{ad}(x)$ on $\mathfrak{c} / \mathfrak{g}(E)$ (respectively on $\mathfrak{p} / \mathfrak{c}, \mathfrak{g} / \mathfrak{p}, \mathfrak{q} / \mathfrak{c}, \mathfrak{g} / \mathfrak{q})$. As $\mathfrak{p}$ and $\mathfrak{q}$ are maximal isotropic subspaces for $B(E)$, we have isomorphisms of $\mathfrak{g}(E)$-modules 
$\mathfrak{g} / \mathfrak{q} \simeq(\mathfrak{q} / \mathfrak{g}(E))^{*}, \mathfrak{q} / \mathfrak{c} \simeq(\mathfrak{p} / \mathfrak{c})^{*}, \mathfrak{g} / \mathfrak{p} \simeq(\mathfrak{p} / \mathfrak{g}(E))^{*}$. Thus we have $\frac{1}{2} q^{\prime}=$ $-\frac{1}{2}(q+c)=-\frac{1}{2}(-q+c)-q=-\frac{1}{2}(p+c)-q=1 / 2 p^{\prime}-q$. In particular, we have $\lambda^{*}\left|\mathfrak{g}(E)-q=\mu^{*}\right| \mathfrak{g}(E)$.

Thus by Lemma 6.3.2, $M(\mathfrak{p}, \lambda, E)$ contains a nonzero vector $v^{\prime}$ such that $u \cdot v^{\prime}=E(u)^{1 / p} \cdot v^{\prime}$ for any $u \in \mathfrak{u}(\mathfrak{q})$ and $x \cdot v^{\prime}=\mu^{*}(x) \cdot v^{\prime}$ for any $x \in \mathfrak{g}(E)$. Hence $U(\mathfrak{g}) \cdot v^{\prime}$ is a quotient of $M(\mathfrak{q}, \mu, E)$. As $M(\mathfrak{p}, \lambda, E)$ and $M(\mathfrak{q}, \mu, E)$ are simple (Weisfeiler-Kac Theorem), they are isomorphic.

(2) Prove the converse. We can assume that $\mathfrak{p}$ and $\mathfrak{q}$ are equal Pukhansky algebras. By Lemma 4.1, there exists $\alpha \in Q(\mathfrak{g})$ such that $\lambda+\alpha$ and $\mu$ coincide over $\mathfrak{p}$. Assume that $M(\mathfrak{p}, \lambda, E)$ and $M(\mathfrak{p}, \mu, E)$ are isomorphic. Set $M=$ $M(\mathfrak{p}, \lambda, E)$, let $I$ be the annihilator of the module $M$, and let $\tau$ be the automorphism of $U(\mathfrak{g})$ induced by $\alpha$. Thus $M$ and $\tau_{*} M$ are isomorphic. Hence $I$ is stabilized by $\tau$. Set $\mathfrak{g}^{\prime}=\operatorname{Ker} \alpha$.

We want to prove that $\mathfrak{g}(E)$ is orthogonal to $\alpha$. Assume that $\alpha(\mathfrak{g}(E)) \neq 0$. Then $B(E)$ and $B(E) \mid \mathfrak{g}^{\prime}$ have the same rank. By the Weisfeiler-Kac Theorem, $M$ is simple as a $U\left(\mathfrak{g}^{\prime}\right)$-module. So we have $U\left(\mathfrak{g}^{\prime}\right)+I=U(\mathfrak{g})$. Hence $\tau$ acts trivially on $U(\mathfrak{g}) / I$. Choose $h \in \mathfrak{g}$ such that $\alpha(h)=1$. So we get that $1=h-\tau(h)$ belongs to $I$, which is impossible. Hence $\mathfrak{g}(E)$ is orthogonal to $\alpha$ and the converse is proved. Q.E.D.

Lemma 6.5. Let $(\mathfrak{a}, \mu, E)$ be in $\mathscr{T}$. Assume that $[\mathfrak{g}(E), \mathfrak{a}] \subset \mathfrak{a}$.

(6.5.1) There exists $\lambda \in \mathfrak{g}^{*}$ such that $\lambda|\mathfrak{a}=\mu, C \lambda| \mathfrak{g}(E)=E \mid \mathfrak{g}(E)$, and $\lambda(u)^{p}=E(x)$ for any $u \in \mathfrak{u}$.

(6.5.2) Let $\mathfrak{p}$ be any Pukhansky polarization at $E$. Then the simple subquotients of $M(\mathfrak{a}, \mu, E)$ are the modules $M(\mathfrak{p},(\lambda+\alpha) \mid \mathfrak{p}, E)$, where $\alpha$ belongs to $\Delta_{\lambda}(\mathfrak{a})$ (the multiplicity of a simple factor in a composition series of $M(\mathfrak{a}, \mu, E)$ is usually > 1).

Proof. (1) Set $\mathfrak{a}^{\prime}=\mathfrak{a}+\mathfrak{g}(E)$. Using Lemma 4.1, it easy to prove that there exists $\nu \in X\left(\mathfrak{a}^{\prime}\right)$ such that $\nu$ extends $\mu$ and $C \nu=E \mid \mathfrak{a}^{\prime}$. Thus $\nu$ and $E^{1 / p}$ coincide over $\mathfrak{u}\left(\mathfrak{a}^{\prime}\right)$. So there exists a linear form $\lambda$ whose restriction to $\mathfrak{a}^{\prime}$ is $\nu$ and whose restriction to $\mathfrak{u}$ is $E^{1 / p}$. Hence (6.5.1) is proved.

Moreover, we have $[\mathfrak{p}, \mathfrak{p}] \subset \mathfrak{u}$. Hence $\lambda \mid \mathfrak{p}$ is a weight of $\mathfrak{p}$. By Lemma 4.3, $\mathfrak{p}$ is a Pukhansky polarization at $\lambda$. Also note that $B(E)=B(\lambda)^{p}$ so the notion of isotropy and orthogonality are the same for $E$ and for $\lambda$. Moreover, for any restricted isotropic subalgebra $\mathfrak{c}$ such that $\mathfrak{c}=\mathfrak{u}+\left(\mathfrak{a}^{\prime} \cap \mathfrak{c}\right)$ we have $C(\lambda \mid \mathfrak{c})=E \mid \mathfrak{c}$.

(2) Again set $\mathfrak{a}^{\prime}=\mathfrak{a}+\mathfrak{g}(E)$. We have $\Delta_{\lambda}\left(\mathfrak{a}^{\prime}\right) \subset \Delta_{\lambda}(\mathfrak{a})$. Moreover, all the elements of $\operatorname{def}(\mathfrak{a}) \backslash \operatorname{def}\left(\mathfrak{a}^{\prime}\right)$ are orthogonal to $\mathfrak{a} \cap \mathfrak{g}(\lambda)$. So we have $\Delta_{\lambda}(\mathfrak{a})=$ $I(\mathfrak{a})+\Delta_{\lambda}\left(\mathfrak{a}^{\prime}\right)$. Hence by Lemma 6.3.4 it suffices to prove the assertion for $\mathfrak{a}^{\prime}$ instead of $\mathfrak{a}$. So now we can assume that $\mathfrak{a}$ contains $\mathfrak{g}(E)$. The proof will follow the same lines as the proof of Lemma 2.4 .

(3) By Lemma 2.6 there exists an integer $n$ and for $0 \leq i \leq n$ some elements $\left(\mathfrak{a}_{i}, \mathfrak{b}_{i}\right)$ of $\mathscr{X}(\lambda)$ such that

(6.5.3) $\mathfrak{a}_{0}=\mathfrak{a}$ and $\mathfrak{b}_{0}=\mathfrak{g}$, 
(6.5.4) $\mathfrak{a}_{n}=\mathfrak{b}_{n}$; in particular $\mathfrak{a}_{n}$ is a Pukhanky polarization of $E$, and

(6.5.5) for $i<n,\left(\mathfrak{a}_{i}, \mathfrak{b}_{i}\right) \mapsto\left(\mathfrak{a}_{i+1}, \mathfrak{b}_{i+1}\right)$ is an admissible move in $\mathscr{X}(\lambda)$.

When $\mathfrak{a}$ is a Pukhansky polarization of $\lambda$ then $\Delta_{\lambda}(\mathfrak{a})$ is orthogonal to $\mathfrak{p}$ and (6.5.2) follows from Lemma 6.4. Hence it suffices to prove the following assertion:

(6.5.6) If $(\mathfrak{a}, \mathfrak{b}) \mapsto\left(\mathfrak{a}^{\prime}, \mathfrak{b}^{\prime}\right)$ is an admissible move in $\mathscr{X}(\lambda)$, the assertion of Lemma 5.6.2 is true for $\mathfrak{a}$ whenever it is true for $\mathfrak{a}^{\prime}$.

For an ineffective move $(\mathfrak{a}, \mathfrak{b}) \mapsto\left(\mathfrak{a}^{\prime}, \mathfrak{b}^{\prime}\right)$ (i.e., a move of type (1.a) or (3.a)) assertion (6.5.6) is obvious.

(4) Let $(\mathfrak{a}, \mathfrak{b}) \mapsto\left(\mathfrak{a}^{\prime}, \mathfrak{b}^{\prime}\right)$ be a move of type (3.b) with $\alpha(\mathfrak{a})=0$ or of type (1.b), (2). Then $\mathfrak{a}$ is an ideal of $\mathfrak{a}^{\prime}$ and we have $I(\mathfrak{a})=I\left(\mathfrak{a}^{\prime}\right), \Delta_{\lambda}(\mathfrak{a})=\Delta_{\lambda}\left(\mathfrak{a}^{\prime}\right)$. Thus assertion (6.5.6) follows from Lemma 6.3.4.

(5) Let $(\mathfrak{a}, \mathfrak{b}) \mapsto\left(\mathfrak{a}^{\prime}, \mathfrak{b}^{\prime}\right)$ be an admissible move of type (3.b) with $\alpha(\mathfrak{a}) \neq 0$ or of type (1.c). Let $F$ be the restriction of $E$ to $\mathfrak{a}^{\prime}$. Define $\mu^{*} \in \mathfrak{a}^{*}$ and $\lambda^{*} \in$ $\mathfrak{a}^{\prime *}$ by $\mu^{*}(g)=\mu(g)+\frac{1}{2} \operatorname{Tr}(\operatorname{ad} g \mid \mathfrak{g} / \mathfrak{a})$ and $\lambda^{*}\left(g^{\prime}\right)=\lambda\left(g^{\prime}\right)+\frac{1}{2} \operatorname{Tr}\left(\operatorname{ad} g^{\prime} \mid \mathfrak{g} / \mathfrak{a}^{\prime}\right)$ for any $g \in \mathfrak{a}, g^{\prime} \in \mathfrak{a}^{\prime}$. Set $N=\operatorname{ind}_{F}\left(\mathfrak{a}, \mathfrak{a}^{\prime}\right) \mu^{*}$. Then the simple subquotients of the $\mathfrak{a}^{\prime}$-module $N$ have dimension 1 and they correspond to weights $\lambda^{*}+s \cdot \alpha \mid \mathfrak{a}^{\prime}$, where $s$ runs over $\mathbf{F}_{p}$. By transitivity of inductions $M(\mathfrak{a}, \mu, E)$ admits a filtration whose subquotients are $M\left(\mathfrak{a}^{\prime},(\lambda+s \alpha) \mid \mathfrak{a}^{\prime}, E\right)$, where $s \in \mathbf{F}_{p}$. Since $\Delta_{\lambda}(\mathfrak{a})=\Delta_{\lambda}\left(\mathfrak{a}^{\prime}\right)+\mathbf{F}_{p} \cdot \alpha$, assertion (6.5.6) is proved in this case.

(6) Let $(\mathfrak{a}, \mathfrak{b}) \mapsto\left(\mathfrak{a}^{\prime}, \mathfrak{b}^{\prime}\right)$ be an admissible move of type (1.d) or (3.c). Set $\mathfrak{k}=\mathfrak{a}+\mathfrak{a}^{\prime}$. Let $F$ be the restriction of $E$ to $\mathfrak{k}$. Define $\mu^{*} \in \mathfrak{a}^{*}$ and $\mu^{\prime}, \mu^{\prime *} \in \mathfrak{a}^{\prime *}$ by $\mu^{*}(g)=\mu(g)+\frac{1}{2} \operatorname{Tr}(\operatorname{ad} g \mid \mathfrak{g} / \mathfrak{a}), \mu^{\prime}\left(g^{\prime}\right)=\lambda\left(g^{\prime}\right)$, and $\mu^{\prime *}\left(g^{\prime}\right)=\lambda\left(g^{\prime}\right)+\frac{1}{2} \operatorname{Tr}\left(\operatorname{ad} g^{\prime} \mid \mathfrak{g} / \mathfrak{a}^{\prime}\right)$ for any $g \in \mathfrak{a}, g^{\prime} \in \mathfrak{a}^{\prime}$. Set $N=\operatorname{ind}_{F}(\mathfrak{a}, \mathfrak{k}) \mu^{*}$ and $N^{\prime}=\operatorname{ind}_{F}\left(\mathfrak{a}^{\prime}, \mathfrak{k}\right) \mu^{\prime *}$. It follows from Lemmas 6.1, 6.2, and 6.3.2 that the $\mathfrak{k}$-modules $N$ and $N^{\prime}$ have dimension $p$ and they are simple and isomorphic. By transitivity of inductions the modules $M(\mathfrak{a}, \mu, E)$ and $M\left(\mathfrak{a}^{\prime}, \mu^{\prime}, E\right)$ are isomorphic. Moreover, we have $\Delta_{\lambda}(\mathfrak{a})=\Delta_{\lambda}\left(\mathfrak{a}^{\prime}\right)$. So assertion (6.5.6) is also proved in this case. Q.E.D.

Lemma 6.6. Let $\phi: X \rightarrow \mathscr{T}$ be a morphism of varieties. For any dense subset $Y$ of $X$ we have $L(X)=\bigcap_{y \in Y} L(\phi(y))$. Moreover, if $X$ is irreducible and if $\phi^{-1} \mathscr{V}$ is dense in $X$ then $L(X)$ is prime.

Proof. Let $M_{\alpha}$ be a collection of $U(\mathfrak{g})$-modules and let $M$ be a submodule of $\prod M_{\alpha}$. We say that $M$ is big enough if for any $\alpha$ the projection $M \rightarrow M_{\alpha}$ is onto. It is clear that if $M$ is big enough, then the annihilator of $M$ is the intersection of annihilators of the $M_{\alpha}$ 's. Let $U$ be any affine dense open subset of $X$ and let $Y$ be any dense subset of $X$. As $Y \cap U$ is dense in $U$, the map $\Gamma(U, \mathscr{M}) \rightarrow \prod_{y \in Y \cap U} L(\phi(y))$ is injective and $\Gamma(U, \mathscr{M})$ is big enough in $\prod_{y \in Y \cap U} L(\phi(y))$. Thus we have $L(U)=\bigcap_{y \in Y \cap U} L(\phi(y))$. So the ideal $L(U)$ does not depend on $U$ and we have $L(X)=\bigcap_{y \in Y} L(\phi(y))$.

Assume now that $X$ is irreducible and $\phi^{-1} \mathscr{V}$ is dense in $X$. Let $I, J$ be two ideals with $I \cdot J \subset L(X)$. Set $A=\{x \in X: I \subset L(\phi(x))\}$ and 
$B=\{x \in X: J \subset L(\phi(x))\}$. As $L(z)$ is a maximal ideal for any $z \in \mathscr{V}$ (Weisfeiler-Kac Theorem), we have $\phi^{-1} \mathscr{V} \subset A \cup B$. Hence $A$ or $B$ is dense in $X$ and one of the two ideals $I$ or $J$ is contained in $L(X)$. Q.E.D.

Lemma 6.7. Let $X$ be a G-invariant irreducible subvariety of $\mathfrak{g}^{*}$. Then we have $I(X)=\bigcap_{A \in \Psi(X)} L(A)$. In particular, $I(X)$ is prime.

Proof. For $\lambda \in X$, let $\mathscr{C}(\lambda)$ be the set of central characters $E$ whose restriction to $\mathfrak{p}(\lambda)$ is $\bar{C} \lambda$ and set $V(\lambda)=\operatorname{ind}^{*}(\mathfrak{p}(\lambda), \mathfrak{g}) \lambda$. Then it is clear that $V(\lambda)$ is big enough in $\prod_{E \in \mathscr{C}(\lambda)} M(\mathfrak{p}(\lambda), \lambda, E)$.

Let $X^{0}$ be an open subset of $X$ as in 3.0. For any $\lambda \in X^{0}$, denote by $m(\lambda)$ the corresponding maximal ideal of $k\left[X^{0}\right]$. The module $M\left(\lambda_{X^{0}}\right)$ is projective as a $k\left[X^{0}\right]$-module and for any point $\lambda \in X^{0}$ we have $M\left(\lambda_{X^{0}}\right) / m(\lambda) \cdot M\left(\lambda_{X^{0}}\right)=$ $V(\lambda)$. Hence $M\left(\lambda_{X^{0}}\right)$ is big enough in $\prod_{\lambda \in X^{0}} V(\lambda)$. Thus $M\left(\lambda_{X^{0}}\right)$ is big enough in $\prod_{\lambda \in X^{0}, E \in \mathscr{X}(\lambda)} M(\mathfrak{p}(\lambda), \lambda \mid \mathfrak{p}(\lambda), E)$. It follows from Lemma 6.6 that $I(X)=L(\Psi(X))$ and that $I(X)$ is prime. Q.E.D.

Lemma 6.8. Let $X$ be a G-invariant irreducible subvariety of $\mathfrak{g}^{*}$, let $\nu \in$ $\mathfrak{g}^{*}$, and let $E$ be any central character of $\mathfrak{g}$ extending $\bar{C} \nu$. Assume that $L(\mathfrak{p}(\nu), \nu \mid \mathfrak{p}(\nu), E)$ contains $I(X)$. Then $\nu$ belongs to $X$.

Proof. Set $\mathfrak{p}=\mathfrak{p}(\nu)$. By Lemma 2.2, all linear forms extending $\nu \mid \mathfrak{p}$ are in the $G$-orbit of $\nu$. Moreover, we have $\nu(x)^{p}=E(x)$ for any $x \in \mathfrak{u}(\mathfrak{p})$. So we can assume that $\nu(x)^{p}=E(x)$ for any $x \in \mathfrak{u}$. In particular, we have $\mathfrak{g}(E)=\mathfrak{g}(\nu)$.

Let $m(C X)$ be the ideal of $O(\mathfrak{g})$ defined by the variety $C X$. By Lemma 6.7, we have $I(X)=\bigcap_{A \in \Psi(X)} L(A)$. Moreover, for any $(\mathfrak{a}, \mu, F) \in \Psi(X)$ we have $L(\mathfrak{a}, \mu, F) \cap O(\mathfrak{g})=m(F)$. Hence we have $I(X) \cap O(\mathfrak{g})=m(C X)$. Hence $D=U(\mathfrak{g}) / I(X)$ is a torsion-free, prime, and finite $k[C X]$-algebra. Let $\mathscr{D}$ be the corresponding $\mathscr{O}_{C X}$-algebra.

Let $\mathscr{M}$ be the canonical vector bundle over $\Psi(X)$ whose fiber over a point $(\mathfrak{a}, \mu, F) \in \Psi(X)$ is $M(\mathfrak{a}, \mu, F)$. Consider $\mathscr{M}$ as a $\left(\mathscr{O}_{\Psi(X)} \otimes U(\mathfrak{g})\right)$-module, and let $\mathscr{J}$ be its annihilator. Set $\mathscr{E}=\mathscr{O}_{\Psi(X)} \otimes U(\mathfrak{g}) / \mathscr{J}$. Note that $\mathscr{E}$ is a torsion-free $\mathscr{O}_{\Psi(X)}$-algebra because $\mathscr{M}$ is torsion-free. The map $\tau: \Psi(X) \rightarrow C X$ is projective and surjective (Lemma 4.6) and the natural map $\pi: \mathscr{D} \rightarrow \tau_{*} \mathscr{E}$ is an extension. Hence, by Lemma 5.2, there exists a maximal ideal $\mathscr{R}$ of $\mathscr{E}$ such that $\pi^{-1} \mathscr{R}=L(\mathfrak{p}, \nu \mid \mathfrak{p}, E)$. Let $m=\mathscr{R} \cap \mathscr{O}_{\Psi_{(X)}}$ be the corresponding point of $\Psi(X)$. Set $m=(\mathfrak{a}, \mu, F)$. By Lemma 5.1, $\mathscr{R}$ is the annihilator of a simple subquotient of $M(\mathfrak{a}, \mu, F)$.

Hence $L(\mathfrak{p}, \nu \mid \mathfrak{p}, E)$ is the annihilator of a simple subquotient of $M(\mathfrak{a}, \mu, F)$ with $(\mathfrak{a}, \mu, F) \in \Psi(X)$. Note that we have $E=F$. Moreover, by Lemma 6.3, we can assume that $(\mathfrak{a}, \mu, E)$ is $G(E)$-invariant.

Lemma 6.5.1 allows us to choose some $\lambda \in \mathfrak{g}^{*}$ such that $\lambda \mid \mathfrak{a}=\mu, C(\lambda \mid \mathfrak{g}(E))$ $=E \mid \mathfrak{g}(E)$, and $\lambda(x)^{p}=E(x)$ for $x \in \mathfrak{u}$. Note that we have $\mathfrak{g}(\lambda)=\mathfrak{g}(\nu)$. So we have $Q_{\lambda}(\mathfrak{a})=Q_{\nu}(\mathfrak{a})$. Set $\Delta=k \otimes \Delta_{\lambda}(\mathfrak{a})=k \otimes \Delta_{\nu}(\mathfrak{a})$ and $\mathfrak{q}=\mathfrak{u}+\mathfrak{g}(\lambda)=\mathfrak{u}+\mathfrak{g}(\nu)$. By Lemma 6.5.2 the simple subquotients of $M(\mathfrak{a}, \mu, E)$ are $M(\mathfrak{p},(\lambda+\alpha) \mid \mathfrak{p}, E)$ 
for some $\alpha \in \Delta$. Thus by Lemma 6.5, there exists some $\alpha \in \Delta$ such that $\lambda+\alpha$ and $\nu$ coincide over $\mathfrak{g}(\nu)$. Moreover, the restrictions to $\mathfrak{u}$ of $\lambda+\alpha$ and $\nu$ are equal to $E^{1 / p} \mid \mathfrak{u}$. By the definition of $Q_{\lambda}(\mathfrak{a}), \Delta$ contains the orthogonal of $\mathfrak{q}$. So we have $\nu=\lambda+\beta$ for some $\beta \in \Delta$.

By hypothesis $(\mathfrak{a}, \mu)$ belongs to $\Theta(X)$. So by Lemma $2.4,\left(\mathfrak{a}, \lambda+\mathfrak{a}^{\perp}\right)$ is contained in $\Sigma(X)$. Hence $X(\mathfrak{a}, \lambda)$ is contained in $X$. Hence by Lemma 2.7, $\lambda+\Delta$ is contained in $X$, i.e., $\nu$ belongs to $X$. Q.E.D.

Lemma 6.9. Let $X, Y$ be G-invariant irreducible subvarieties of $\mathfrak{g}^{*}$. Assume that $I(X) \subset I(Y)$. Then $X$ contains $Y$.

Proof. Let $\nu$ be any element of $Y_{\text {reg }}^{0}$ and let $E$ be any central character of $\mathfrak{g}^{*}$ extending $\bar{C} \nu$. By Lemma 6.7, $L(\mathfrak{p}(\nu), \nu \mid \mathfrak{p}(\nu), E)$ contains $I(Y)$. Hence $L(\mathfrak{p}(\nu), \nu \mid \mathfrak{p}(\nu), E)$ contains $I(X)$. So by Lemma 6.8, $\nu$ belongs to $X$. This proves $Y_{\text {reg }}^{0} \subset X$. As $Y_{\text {reg }}^{0}$ is dense in $Y$, we have $Y \subset X$. Q.E.D.

Proof of the main Theorem. Lemmas 3.2 and 6.9 imply the Theorem.

Proof of Theorem 4.7. (1) By Lemma 4.6, $C X$ is irreducible. Let $E$ be a central character and let $\mathfrak{p}$ be a restricted subalgebra of $\mathfrak{g}$.

First assume $E \in C X$ and that $\mathfrak{p}$ is a Pukhansky polarization at $E$. By definition there exists $(\mathfrak{a}, \mu, F) \in \Psi(X)$ with $F=E$. By Lemma 6.3 we can assume that $\mathfrak{a}$ is $G(E)$-invariant. By Lemma 6.5.1 there exists a linear form $\lambda$ extending $\mu$ on $\mathfrak{a}$ and $E^{1 / p}$ on $\mathfrak{u}$ such that $C(\lambda \mid \mathfrak{g}(E))=E \mid \mathfrak{g}(E)$. Thus we have $C(\lambda \mid \mathfrak{p})=E \mid \mathfrak{p}$. Moreover, by Lemma $4.3, \mathfrak{p}$ is a polarization at $\lambda$ and by Lemma $2.4, \lambda$ belongs to $X$.

Let $\lambda \in X$ be such that $\mathfrak{p}$ is a Pukhansky polarization at $\lambda$ and $C(\lambda \mid \mathfrak{p})=$ $E \mid \mathfrak{p}$. Then we similarly prove that $E$ belongs to $C X$.

Moreover, it follows from Lemma 6.7 that $C X$ is defined by the ideal $I(X) \cap$ $O(\mathfrak{g})$. Hence assertions (4.7.1) and (4.7.2) are proved.

(2) It is clear that $\operatorname{dim} X=\operatorname{dim} \Psi(X)=\operatorname{dim} C X$. Moreover, the indexes of $X$ and $C X$ are the same. As $X$ (respectively $C X$ ) is the closure of an orbit if and only if $\operatorname{dim} X=2 \cdot \operatorname{ind}(X)$ (respectively $\operatorname{dim} C X=2 \cdot \operatorname{ind}(C X)$ ), assertions (4.7.3) and (4.7.4) follow.

(3) Assertion 4.7.5 follows easily from Lemma 4.5. Q.E.D.

Actually the main Theorem can be extended for fields $k$ of finite characteristics. Let $\operatorname{Spec}^{G} S \mathfrak{g}$ and $\operatorname{Spec}^{G} U(\mathfrak{g})$ be the set of $G$-invariant prime ideals of $S \mathfrak{g}$ and $U(\mathfrak{g})$.

Theorem 6.10. The Dixmier map I: $\operatorname{Spec}^{G} S \mathfrak{g} \rightarrow \operatorname{Spec}^{G} U(\mathfrak{g})$ is bicontinuous. Proof. Let $P \in \operatorname{Spec}^{G} U(\mathfrak{g})$, set $M=P \cap O(\mathfrak{g})$, let $Y$ be the subvariety of $\left(F_{*} \mathfrak{g}\right)^{*}$ defined by $M$, let $K$ be an algebraic closure of $k(Y)$, and let $C^{-1}(Y)$ be the set of $G$-invariant irreducible subvarieties $X$ of $\mathfrak{g}^{*}$ such that $C X=$ $Y$. It follows from the theorem of Weisfeiler and Kac applied to $\mathfrak{g} \otimes K$ that all the $G$-invariant prime ideals $J$ of $U(\mathfrak{g})$ such that $J \cap O(\mathfrak{g})=M$ are 
conjugated under $Q(\mathfrak{g})$. For any $X \in C^{-1} Y$, the ideal $I(X)$ is prime and we have $I(X) \cap O(\mathfrak{g})=M$ (Lemmas 4.7.1 and 6.7). Hence by Lemma 4.7.5, we have $P=I(X)$ for some $X \in C^{-1} Y$. Hence $I$ is onto. The continuity of $I$ follows from Lemma 6.5 and the usual proof [CD, CV]. The injectivity of $I$ and the continuity of $I^{-1}$ follow from Lemma 6.9. Q.E.D.

\section{ACKNOWLEDGMENTS}

We would like to thank F. du Cloux [D], M. Duflo, R. Rentschler, and R. Wilson for helpful discussions. M. Duflo found an error in an earlier manuscript. We heartily thank the referee and S. Evans for their suggestions.

\section{BIBLIOGRAPHY}

[AK] L. Auslander and B. Kostant, Polarization and unitary representations of solvable Lie groups, Invent. Math. 14 (1971), 255-354.

[B] P. Bernat et. al., Représentations des groupes de Lie résolubles, Dunod, Paris, 1972.

[BGR] W. Borho, P. Gabriel, and R. Rentschler, Primideale in Einhullenden auflosbarer Liealgebren, Lecture Notes in Math., vol. 357, Springer-Verlag, Berlin, 1973.

[Ca] P. Cartier, Question de rationalité en géométrie algébrique, Bull. Soc. Math. France 86 (1958), 177-251.

[C] N. Conze, Espace des idéaux primitifs de l'algèbre enveloppante d'une algèbre de Lie nilpotente, J. Algebra 34 (1975), 440-450.

[CD] N. Conze and M. Duflo, Sur l'algèbre enveloppante d'une algèbre de Lie résoluble, Bull. Soc. Math. France 94 (1970), 201-208.

[CV] N. Conze and M. Vergne, Idéaux primitifs des algèbres enveloppantes des algèbres de Lie résolubles, C. R. Acad. Sci. Paris Sér. I. Math. 272 (1971), 985-988.

[DI] P. Deligne and C. Illusie, Revétement modulo $p^{2}$ et scindage du complexe de De Rham, Inv. Math. 89 (1987), 247-270.

[Di $\left.{ }_{1}\right]$ J. Dixmier, Représentations irréductibles des algèbres de Lie nilpotentes, An. Acad Brasil. Ciênc. 35 (1963), 491-519.

$\left[\mathrm{Di}_{2}\right] \_$, Représentations irréductibles des algèbres de Lie résolubles, J. Math. Pures Appl. 45 (1966), 1-66.

[Di $\left.{ }_{3}\right]$ _ Algèbres enveloppantes, Gauthier-Villars, Paris, 1974.

[D] F. du Cloux, Représentations de longueur finie des groupes de Lie résolubles, Mem. Amer. Math. Soc. No. 407 (1989).

[Du $\left.{ }_{1}\right]$ M. Duflo, Sur les représentations irréductibles des algèbres de Lie contenant un idéal nilpotent, C. R. Acad. Sci. Paris Sér. I Math. 270 (1970), 504-506.

$\left[\mathrm{Du}_{2}\right] \ldots$ Caractères des groupes et algèbres de Lie résolubles, Ann. Sci. École. Norm. Sup. 5 (1972), 71-120.

[J] N. Jacobson, Lie algebras, Interscience, New York, (1962).

[Ki] A. A. Kirillov, Unitary representations of nilpotent Lie groups, Uspekhi Mat. Nauk 17 (1962), 57-110. (Russian)

$\left[\mathrm{M}_{1}\right]$ O. Mathieu, Une formule sur les opérateurs différentiels en caractéristique non nulle, $\mathrm{C} . \mathrm{R}$. Acad. Sci. Paris Sér. I Math. 304 (1987), 405-406.

$\left[\mathrm{M}_{2}\right]$ - Classification of Harish-Chandra modules for the Virasoro algebra, Invent. Math. (to appear).

[P] C. Procesi, Rings with polynomial identities, Marcel Dekker, New York, 1973. 
[Q] D. Quillen, On the endomorphism ring of a simple module over an enveloping algebra, Proc. Amer. Math. Soc. 21 (1969), 171-172.

[R] R. Rentschler, L'injectivité de l'application de Dixmier, Invent. Math. 23 (1974), 49-71.

[SF] H. Strade and R. Farnsteiner, Modular Lie algebras and their representations, Marcel Dekker, New York, 1988.

[WK] B. J. Weisfeiler and V. G. Kac, The irreducible representations of Lie p-algebras, Functional Anal. Appl. 5 (1971), 111-117.

École Normale Supérieure, 45 Rue d'Ulm, 75005 Paris, France

Current address: Rutgers University, Hill Center, New Brunswick, New Jersey 08903 